Japan. J. Math.

Vol. 5, No. 1, 1979

\title{
Finite groups with a standard subgroup isomorphic to $U_{4}\left(2^{n}\right)$
}

\author{
By Izumi Miуамото
}

(Received Feb. 10, 1977)

Let $K$ be a subgroup of a finite group $G$. If $K$ has even order while $K \cap K^{g}$ has odd order for any $g \in G-N_{G}(K)$, then $K$ is said to be tightly embedded in $G$. Furthermore, a quasisimple subgroup $L$ of $G$ is said to be a standard subgroup if $K=C_{G}(L)$ is tightly embedded in $G, N_{G}(K)=N_{G}(L)$ and $\left[L, L^{g}\right] \neq 1$ for any $g \in G$. In this paper we prove the following theorem.

THEOREM. Let $G$ be a finite group and let $L$ be a standard subgroup of G. Assume that $L / Z(L) \simeq U_{4}(q)$, where $q=2^{n}>2$. Furthermore assume that $C_{G}(L)$ has a cyclic Sylow 2-subgroup. Let $X$ be the normal closure of $L$ in $G$. Then one of the following holds:

(1) $L O(G) \triangleleft G$

(2) $X \simeq L_{4}\left(q^{2}\right)$

(3) $X \simeq U_{4}(q) \times U_{4}(q)$.

After the fundamental work of Aschbacher [2], much work has been done toward the classification of finite groups with a standard subgroup of known type (e.g. [5], [12], [16], [20], [21]). For details we refer the readers to [12]. As stated in the theorem above, the result of this paper classifies the groups with a standard subgroup isomorphic to $U_{4}\left(2^{n}\right)$ with $2^{n}>2$ whose centralizer has a cyclic Sylow 2-subgroup.

In order to prove the theorem we may assume that $O(G)=1$ (cf. Section 6). Then provided that $L \rtimes G$, our aim is to obtain that $\left\langle L^{G}\right\rangle \simeq L \times L$ or $L_{4}\left(q^{2}\right)$. The method used in this paper is essentially a careful analysis of 2local subgroups of $G$ which heavily depends on the structure of 2-local subgroups of $U_{4}(q)$. Let $A_{1}$ and $A_{2}$ be the largest normal 2-subgroups of the maximal parabolic subgroups of $L$. Then a major part of the proof is devoted to constructing the 2-subgroups $F_{1}$ and $F_{2}$ such that $N_{L}\left(A_{i}\right)^{\prime}<N\left(F_{i}\right)$ (cf. (3.5), (3.9)). Next, applying the previous classification theorem [16] to the groups $N_{G}\left(F_{i}\right) / F_{i}$, we construct the subgroups $L_{i}$ of $N_{G}\left(F_{i}\right), i=1,2$ (cf. (3.12)). Here two cases occur:

(1) $L_{1} / F_{1} \simeq L_{2}\left(q^{2}\right) \times L_{2}\left(q^{2}\right), L_{2} / F_{2} \simeq L_{2}\left(q^{2}\right) ;$ 
(2) $\quad L_{1} / F_{1} \simeq L_{2}\left(q^{2}\right) \times L_{2}\left(q^{2}\right), L_{2} / F_{2} \simeq L_{2}(q) \times L_{2}(q)$.

Case (1) is treated in Section 4 and Case (2) in Section 5. We note that $L_{1}$ is obtained as a normal closure of $N_{L}\left(A_{1}\right)^{\prime}$ in $N\left(F_{1}\right)$ while $L_{2}$ is not obtained in the same way and some fusion argument is needed to prove $L_{2} \triangleleft N\left(F_{2}\right)$.

The author would like to express his hearty thanks to Dr. Kensaku Gomi. The general line of the proof is shown by him in his paper [12] on which the author greatly depends. Also the author is grateful to Dr. Hiromichi Yamada for providing a lemma appearing as (1.6) in the present paper which contributes to the simplification of the argument on 2-groups in Section 3.

Our notation is standard and we refer the readers to [13] except possibly the following. If $X$ is a 2-group,

$\mathscr{E}(X)=$ the set of the maximal elementary abelian subgroups of $X$,

$\operatorname{Jr}(X)=$ the subgroup generated by the elementary abelian subgroup of $X$ of maximal rank.

By $J(X)$ we denote the usual Thompson subgroup generated by the abelian subgroups of $X$ of maximal order. For any finite group $G$,

$E(G)=$ the maximal normal semisimple subgroup of $G$,

$G^{(\infty)}=$ the final term of the derived series of $G$,

$G^{i}=\left\langle g^{i} \mid g \in G\right\rangle$ for an integer $i$,

$I(X)=$ the set of the involutions of any subset $X$ of $G$.

If $X$ and $Y$ are subgroups of a group,

$X * Y=a$ central product of $X$ and $Y$,

$X \cdot Y=a$ semidirect product of $X$ by $Y$.

For subsets $X$ and $Y$ of a group,

$$
X^{Y}=\left\{y^{-1} x y \mid x \in X, y \in Y\right\} .
$$

If $X$ acts on a set $\Omega$, then $X^{\Omega}$ denotes the image of the permutation representation of $X$ on $\Omega$. By Goldschmidt groups we mean quasisimple groups called groups of type I and II in [10].

\section{$\S 1 . \quad$ Preliminaries}

(1.1) Let $\langle t\rangle \times A$ be a normal 2-subgroup of a group $H$ and suppose $\langle t\rangle \times A$ is elementary abelian, $\left|H: C_{H}(t)\right|=$ even and $C_{H}(t) \cap N_{H}(A)$ acts transitively on $A^{\#}$ by conjugation. Let $\bar{H}=H / C_{H}(\langle t\rangle A)$. Furthermore suppose 
that $\overline{C_{H}(t)}$ has a cyclic normal subgroup $\bar{J}$. Then $\overline{C_{H}(A)}$ is elementary abelian of order $|A|$ and $t^{H} t^{C(A)}=t A$.

Proor. We will consider the action of $H$ on $t^{H}$. Then $t^{H}=\{t\} \cup A^{\#}$ or $t A$. In any case $H$ is doubly transitive on $t^{H}$ and contains a cyclic normal subgroup in its one-point-stabilizer. Hence by Theorem 3 of [1] either $\bar{H}$ has a regular normal subgroup on $t^{H}$ or $\left\langle\bar{J}^{H}\right\rangle \simeq L_{2}(r)$ for some prime $r$. Suppose that the former holds and let $M$ be the preimage of the regular normal subgroup and let $S$ be a Sylow 2-subgroup of $M$. Then $Z(M) \cap\langle t\rangle A$ $=Z(S) \cap\langle t\rangle A$. Hence $Z(M) \cap\langle t\rangle A \neq 1 . \quad C_{H}(t) \cap N_{H}(A)$ acts on $Z(M) \cap\langle t\rangle A$ and this action yields that $Z(M) \cap\langle t\rangle A=A$ and $t^{H}=t A$. Suppose that the latter holds. Then, since $\left\langle\bar{J}^{H}\right\rangle$ acts also on $(\langle t\rangle A)^{\sharp}-t^{H}$, we get $\left\langle\bar{J}^{H}\right\rangle \simeq L_{2}(7)$ and $\langle t\rangle A$ has order 16. If $t^{H}=t A$, then $\left\langle\bar{J}^{H}\right\rangle$ is embedded in the parabolic subgroup of $G L_{4}(2)$ leaving the subspace $A$ invariant. Then by a property of Levi decomposition (cf. [6]) $\left\langle\bar{J}^{H}\right\rangle$ has a fixed point on $t A$, which is a contradiction. If $t^{H}=\{t\} \cup A^{\#}$, then a two-point-stabilizer of $t A^{\#}$ in $\left\langle\bar{J}^{H}\right\rangle$, which is a fours group, stabilizes a point on $\{t\} \cup A^{\sharp}$. But a Sylow 2-subgroup of $L_{2}(7)$ acts regularly on $\{t\} \cup A^{\sharp}$. This contradiction completes the proof.

(1.2) Let $q=2^{n}$. Suppose that $q^{4}-q^{3}+q^{2}-q+1=p^{m}$ for some prime $p$. Then $m=1$.

Proof. We prove by way of contradiction. First suppose that $m=2 m_{1}$ $+1, m_{1} \geq 1$. Then

$$
q\left(q^{3}-q^{2}+q-1\right)=(p-1)\left(p^{2 m_{1}}+p^{2 m_{1}-1}+\cdots+1\right) .
$$

Since $q=2^{n}, q$ divides $p-1$. This yields that $m_{1}=1$. Put $p-1=q s$ for some integer $s$. Then

$$
q^{3}-q^{2}+q-1=s\left((q s+1)^{2}+(q s+1)+1\right) .
$$

Hence

$$
q\left(q^{2}-q+1\right)=s^{3} q^{2}+3 s^{2} q+3 s+1 .
$$

Then we have $q \mid 3 s+1$ and also $q \geq s^{3}+1$, which yield that $s=1$. So $q=2$ or 4. Then by direct calculation we can easily derive a contradiction in each case.

Next suppose that $m=2 m_{1}$. Then

$$
q\left(q^{3}-q^{2}+q-1\right)=\left(p^{m_{1}}+1\right)\left(p^{m_{1}}-1\right) .
$$

Hence $p^{m_{1}}-1$ or $p^{m_{1}}+1=\frac{1}{2} q s$ for some odd integer $s$. Then

$$
q\left(q^{2}-q+1\right)=\frac{1}{4} q s^{2}+(1 \pm s) .
$$


Now by direct calculation we may assume that $q \geq 8$. Hence $s \pm 1=\frac{1}{4} q r$ for some integer $r$. Then

$$
\left(64-r^{2}\right) q^{2}+(-64 \pm 8 r) q+48 \mp 16 r=0 .
$$

In either case we have $r \leq 8$. Then checking for each $r, 0 \leq r \leq 8$, we can easily obtain a contradiction.

(1.3) Let $E$ be a normal 2-subgroup of a group $H$. Suppose that $E$ is elementary abelian of order $q^{2} . \quad$ Set $\bar{H}=H / C_{H}(E)$ and suppose that $\bar{H}$ has an abelian normal subgroup $\bar{J}$ which acts on $E^{\#}$ transitively. Let $t$ be an involution in $H-C_{H}(E)$ and let $\bar{x}$ be an element of $\bar{J}$. Then $\bar{x}^{t}=\bar{x}^{q}$.

Proof. We will define multiplication and addition + in $E$ and show that $(E,+, \cdot)$ is a field of $q^{2}$ elements. Name the identity element of $E 0$ and any one element of $E^{\#} 1$. Define multiplication in $E$ as follows. Let $a \cdot 0=0$ for all $a \in E$. For $b \in E, b \neq 0$, there exists a unique element $\bar{u}$ of $\bar{J}$ such that $1^{\bar{u}}=b$, since $\bar{J}$ is regular on $E^{\#}$. Then let $a \cdot b=a^{\bar{u}}$ for $a \in E$. Thus $0 \cdot b=0$. If $1^{\bar{u}}=b$ and $1^{\bar{v}}=c$ for $\bar{u}, \bar{v} \in \bar{J}$, then $1^{\bar{u} \vec{v}}=b^{\bar{v}}$, and $(a \cdot b) \cdot c=$ $\left(a^{\bar{u}}\right) \cdot c=a^{\bar{u} \vec{v}}=a \cdot 1^{\bar{u} \bar{v}}=a \cdot b^{\bar{v}}=a \cdot(b \cdot c)$. Clearly $a \cdot 1=a$, so 1 is a multiplicative identity of $(E,+, \cdot)$. If $1^{\bar{u}}=a$ and $1^{\bar{u}-1}=d$ for $\bar{u} \in \bar{J}$, then $a \cdot d=a^{\bar{u}-1}=1^{\bar{u} \bar{u}^{-1}}$ $=1$. Also $b \cdot c=1^{\bar{u} \bar{v}}=1^{\bar{v} \bar{u}}=c \cdot b$. Hence $E-0$ is an abelian group under the multiplication.

Next define addition as follows. $a+b=a b$ for $a, b \in E$. Then $0+a=$ $a+0=a$, since 0 is defined to be the identity of $E$. Let $a, b$ and $c$ be in $E$. If $c=0$, then $(a+b) \cdot c=0=a \cdot c+b \cdot c$. Now assume that $c \neq 0$ and as above we put $1^{\bar{u}}=c$. Then we have

$$
(a+b) \cdot c=(a+b)^{\bar{u}}=(a b)^{\bar{u}}=a^{\bar{u}} b^{\bar{u}}=(a \cdot c)(b \cdot c)=a \cdot c+b \cdot c .
$$

Thus it is established that $(E,+, \cdot)$ is a finite field of $q^{2}$ elements.

Suppose that $h \in H$ such that $0^{\bar{h}}=0$ and $1^{\bar{h}}=1$. Let $1^{\bar{u}}=a$ and let $1^{\bar{v}}=b$. Then

$$
\begin{aligned}
& (a+b)^{\bar{h}}=(a b)^{\bar{h}}=a^{\bar{h}} b^{\bar{h}}=a^{\bar{h}}+b^{\bar{h}} \\
& (a \cdot b)^{\bar{h}}=a^{\bar{v} \bar{h}}=1^{\bar{u} \overline{\hat{n}}}=1^{\bar{h}-1 \bar{u} \bar{v} \bar{h}}=1^{\bar{h}-1 \bar{\imath} \bar{h}} \cdot 1^{\bar{h}-1 \bar{v} \bar{h}}=1^{\bar{u} \bar{h}} \cdot 1^{\bar{v} \bar{h}}=a^{\bar{h}} \cdot b^{\bar{h}} .
\end{aligned}
$$

Thus $h \in \operatorname{Aut}((E,+, \cdot))$. So $a^{\bar{\hbar}}=\underbrace{a \cdot a \cdots a}_{2^{i}}$ for any $a \in E$ and for some integer

$i$. Put $j=2^{i}$. For any $h \in H$ there exists $\bar{u} \in \bar{J}$ such that $0^{\bar{u} \tilde{h}}=0$ and $1^{\bar{\imath} \hbar}=1$. Then $1^{\bar{x} \bar{h} \bar{h}}=1^{\bar{x}^{j}}$ for any $\bar{x} \in \bar{J}$. On the other hand $1^{\bar{x} \bar{h} \bar{h}}=1^{(\bar{u} \bar{h})-1 \bar{x} \bar{u}}=1^{\bar{h}-1 \bar{x} \bar{h}}=1^{\bar{x}^{\bar{h}}}$. Thus $1^{\bar{x}^{\bar{h}}}=1^{\bar{x}^{j}}$ and so $\bar{x}^{h}=\bar{x}^{j}$. Hence in particular if $t \in H-C_{H}(E)$ is an involution, then $\bar{x}^{t}=\bar{x}^{q}$. 
(1.4) Let $V$ be an elementary abelian group of order $2^{2 m}$ for some integer $m$. Let $f$ be an element of order $2^{i}$ which acts on $V$. Suppose that $\left|C_{V}\left(f^{2^{i-1}}\right)\right|$ $=2^{m}$. Then any element $x$ in $f^{j} V$ whose order is equal to $\left|f^{j}\right|$ is conjugate to $f^{j}$ in $V\langle f\rangle$.

Proof. If $|x|=2$, then the assertion is easily checked. Suppose that $|x|>2$. Take $v \in V$ such that $x=f^{j} v$. Then by induction we may assume that there exists an element $u$ in $V$ such that $x^{2 u}=f^{2 j}$. This implies that $\left[v, f^{j}\right]=\left[u, f^{2 j}\right]=\left[u, f^{j}, f^{j}\right]$. Hence $v\left[u, f^{j}\right] \in C_{V}\left(f^{j}\right)$. Since $C_{V}\left(f^{j}\right) \leq C_{V}\left(f^{2 i-1}\right)=$ $\left[V, f^{2 i-1}\right] \leq\left[V, f^{j}\right]$ by the assumption, we have $v\left[u, f^{j}\right]=\left[w, f^{j}\right]$ for some $w \in V$. Then $v=\left[u w, f^{j}\right]$ and consequently $x^{u w}=f^{j}$.

The following is the well known (generalized) "Thompson transfer lemma" and the proof is omitted.

(1.5) Let $S$ be a Sylow 2-Subgroup of a group $G$ and let $R$ be a subgroup of $S$ such that $S^{\prime} \leq R$. Suppose that $x$ is an involution of $S-R$ such that $x^{G} \cap S \subseteq x R$. Then $x \notin O^{2}(G)$.

The following is due to H. Yamada [20] (2.4).

(1.6) Let $X$ be a $p^{\prime}$-group acting on a p-group $A$. Suppose that $C_{A}(X)$ $\unlhd A$. Then $A=C_{A}(X) *[A, X]$.

\section{§2. Properties of $U_{4}(q)$}

In this section we fix the notation for various subgroups of $L \simeq U_{4}(q)$, $q=2^{n}>2$, and obtain some basic properties about them and the automorphism group of $L$, which are required in the following sections.

We identify the elements of $L$ with $4 \times 4$ matrices $x$ with entries in $G F\left(q^{2}\right)$ satisfying the conditions

$$
x^{*} r x=r \quad \text { and } \quad \operatorname{det}(x)=1
$$

where $r=\left(\begin{array}{c}1 \\ 1 \\ 1\end{array}\right)$ and $x^{*}$ denotes the adjoint matrix of $x$ conjugated by the involutive automorphism of $G E\left(q^{2}\right)$. L has order $q^{6}\left(q^{2}-1\right)\left(q^{3}+1\right)\left(q^{4}-1\right)$ (see for example [7, p. 8]). For $a \in G F\left(q^{2}\right)$ set $\bar{a}=a^{q}$ and denote by $G E\left(q^{2}\right)^{\times}$the multiplicative subgroup of $G F\left(q^{2}\right)$. Denote by $P$ the subgroup of $L$ consisting of the matrices 


$$
\left(\begin{array}{cccc}
1 & & & \\
a & 1 & & \\
c & b & 1 & \\
d & \bar{c}+\bar{a} b & \bar{a} & 1
\end{array}\right)
$$

where $b \in G F(q)$ and $d+\bar{d}+a \bar{c}+\bar{a} c=0$. Then $P$ is a Sylow 2-subgroup of $L$. We define the following subgroups of $P$ :

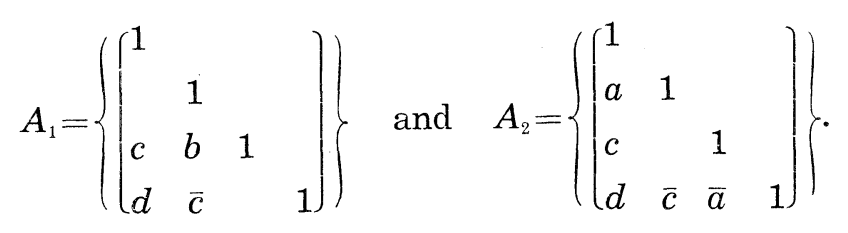

Then $A_{1}$ is elementary abelian. Moreover we have

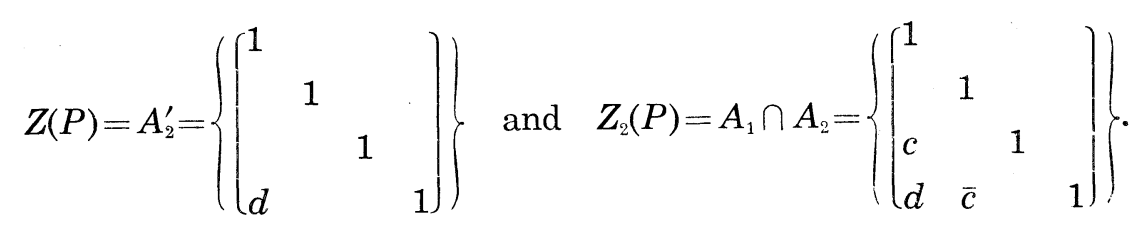

Denote by $I$ the subgroup of $L$ consisting of the matrices

$$
\left\{\begin{array}{llll}
a & & & \\
& b & & \\
& & \bar{b}^{-1} & \\
& & & \bar{a}^{-1}
\end{array}\right)
$$

where $a b \bar{a}^{-1} \bar{b}^{-1}=1$. Furthermore define

$$
\left.I_{1}=\left\{\mid \begin{array}{llll}
a & & \\
& a & \\
& & a^{-1} & \\
& & & a^{-1}
\end{array}\right) \mid a \in G F(q)^{\times}\right\}
$$

and

$$
I_{2}=\left\{\left(\left\{\begin{array}{llll}
a^{2} & & & \\
& a^{q-1} & & \\
& & a^{q-1} & \\
& & & \bar{a}^{-2}
\end{array}\right) \mid a \in G F\left(q^{9}\right)^{\times}\right\},\right.
$$


which are subgroups of $I$.

Denote by $N_{1}$ the subgroup consisting of the matrices

$$
\left\{\begin{array}{cccc}
s & t & & \\
u & v & & \\
& & \bar{s} & \bar{t} \\
& & \bar{u} & \bar{v}
\end{array}\right\},
$$

where $\left(\begin{array}{ll}s & t \\ u & v\end{array}\right) \in S L_{2}\left(q^{2}\right)$. Denote by $N_{2}$ the subgroup consisting of the matrices

$$
\left(\begin{array}{llll}
1 & & & \\
& s & t & \\
& u & v & \\
& & & 1
\end{array}\right),
$$

where $\left(\begin{array}{ll}s & t \\ u & v\end{array}\right) \in S L_{2}(q)$. Finally define

$$
\begin{aligned}
& r_{1}=\left(\begin{array}{llll}
1 & 1 & & \\
& & & 1
\end{array}\right), \quad r_{2}=\left(\begin{array}{llll}
1 & & & \\
& & 1 & \\
& 1 & & \\
& & 1 &
\end{array}\right], \\
& z_{1}=\left(\begin{array}{llll}
1 & & & \\
& 1 & & \\
& & 1 & \\
1 & & & 1
\end{array}\right), \quad z_{2}=\left(\begin{array}{llll}
1 & & & \\
& 1 & & \\
1 & & 1 & \\
& 1 & & 1
\end{array}\right) .
\end{aligned}
$$

Let $A=\operatorname{Aut}(L)$. Then $A$ is a semidirect product of $L$ by a cyclic subgroup generated by a field automorphism $f$ of order $2 n$ which acts on $L$ canonically. Let $u \in I(\langle f\rangle)$ and let $\tilde{P} \in S y l_{2}(A)$ such that $\langle u, p\rangle \leq \tilde{P}$.

Most of the results of this section are obtained by direct calculation and the proofs are omitted or given briefly.

(2.1) (1) $\quad N_{L}(P)=P I$.

(2) $Z(P)=C_{P}\left(I_{2}^{r_{1}}\right), Z_{2}(P)=Z(P) \cdot C_{P}\left(I_{1}^{r_{2}}\right), A_{i}=Z_{2}(P) \cdot C_{P}\left(I_{3-i}\right), P=A_{i} \cdot C_{P}\left(I_{i}\right)$, $i=1,2$.

(3) I acts transitively on $C_{P}\left(I_{i}\right)^{\#}$ and on $C_{P}\left(I_{i}^{r_{3-i}}\right)^{\#}, i=1,2$

(4) $N_{L}\left(A_{i}\right)=A_{i} \cdot\left(I_{i} \times N_{i}\right), i=1,2$.

(5) $N_{L}(Z(P))=N_{L}\left(A_{2}\right)$ and $C_{L}(Z(P))=A_{2} \cdot\left(I_{2}^{q-1} \times N_{2}\right)$. 
(2.2) (1) $N_{1}$ acts on $A_{1}$ irreducibly and $I_{1} \times N_{1}$ has just two orbits $z_{1}^{I_{1} N_{1}}$ and $z_{2}^{I_{1} N_{1}}$ on $A^{\#}$ whose lengths are $(q-1)\left(q^{2}+1\right)$ and $(q-1) q\left(q^{2}+1\right)$ respectively. In particular $A_{1} N_{1}$ is perfect. Also it holds that $\left|z_{1}^{N_{1}}\right|=(q-1)\left(q^{2}+1\right)$ and $\left|\boldsymbol{z}_{2}^{N_{1}}\right|=q\left(q^{2}+1\right)$.

(2) $\quad A_{2} N_{2}$ is perfect. Set $\overline{N_{L}(Z(P))}=N_{L}(Z(P)) / Z(P)$. Let $x_{1} \in I\left(A_{2}-Z(P)\right)$ and let $x_{2} \in A_{2}^{\sharp}-I\left(A_{2}\right)$. Then $\left|\bar{x}_{1}^{I_{2} N_{2}}\right|=(q+1)\left(q^{2}-1\right)$ and $\left|\bar{x}_{2}^{I_{2} N_{2}}\right|=(q-1) q\left(q^{2}-1\right)$.

(3) $C_{L}\left(z_{1}\right)=C_{L}(Z(P))$ and $C_{N_{1}}\left(z_{2}\right)=C_{N_{1}}(u) \simeq S L_{2}(q)$. Moreover $C_{L}\left(z_{2}\right)^{\prime}=$ $\left(A_{1} \cap C(u)\right) C_{N_{1}}(u)$ is perfect.

Proof. By Lemma $4 \mathrm{~B}$ of [8] $A_{1}$ is an irreducible $N_{1}$-module. By calculation we have

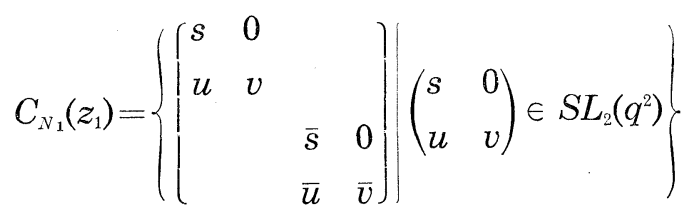

and

$$
C_{N_{1}}\left(z_{2}\right)=C_{N_{1}}(u)=\left\{\left(\left\{\begin{array}{llll}
s & t & & \\
u & v & & \\
& & s & t \\
& u & v
\end{array}\right) \mid\left(\begin{array}{ll}
s & t \\
u & v
\end{array}\right) \in S L_{2}(q)\right\}\right.
$$

Also

$$
C_{I_{1} N_{1}}\left(z_{1}\right)=\left\{\left(\begin{array}{cccc}
s & 0 & & \\
u & v & & \\
& & \bar{s} & 0 \\
& & \bar{u} & \bar{v}
\end{array}\right)\left(\begin{array}{ccc}
1 & & \\
& a & \\
& & a^{-1}
\end{array}\right)\left(\begin{array}{ll}
s & 0 \\
u & v
\end{array}\right) \in S L_{2}\left(q^{2}\right), a \in G F(q)^{\times}\right\}
$$

and

$$
C_{I_{1} N_{1}}\left(z_{2}\right)=\left\{\left(\left\{\begin{array}{cccc}
s & t & & \\
u & v & & \\
& & s & t \\
& & u & v
\end{array}\right) \mid\left(\begin{array}{ll}
s & t \\
u & v
\end{array}\right) \in S L_{2}(q)\right\}\right.
$$

Hence $\left|z_{1}^{I_{1} N_{1}}\right|=(q-1)\left(q^{2}+1\right)$ and $\left|z_{2}^{I_{1} N_{1}}\right|=(q-1) q\left(q^{2}+1\right)$. Since $(q-1)\left(q^{2}+1\right)$ $+(q-1) q\left(q^{2}+1\right)=q^{4}-1,(1)$ holds.

By calculation $P^{\prime}=Z_{2}(P)$. Since $r_{2} \in N_{2}$ and since $A_{2}=Z_{2}(P) Z_{2}(P)^{r_{2}}$, we have $A_{2} \leq\left(A_{2} N_{2}\right)^{\prime}$. Which implies that $A_{2} N_{2}$ is perfect. Similarly we have 


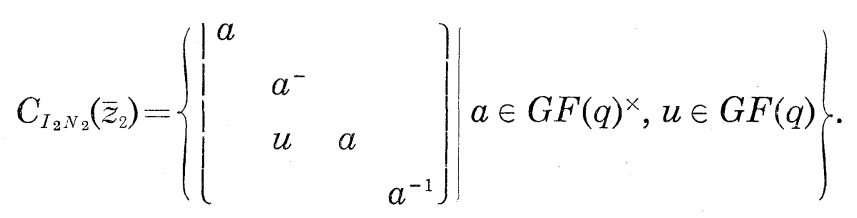

Hence $\left|\bar{z}_{2}^{I_{2} N_{2}}\right|=(q+1)\left(q^{2}-1\right)$. Let $x=\left(\begin{array}{llll}1 & & & \\ 1 & 1 & & \\ c & & 1 & \\ d & \bar{c} & 1 & 1\end{array}\right), c \neq \bar{c}$. Then

$$
\begin{aligned}
& C_{I_{2} N_{2}}(\bar{x})=\left\{\begin{array}{llll}
1 & & & \\
& s & t & \\
& u & v & \\
& & & 1
\end{array}\right\}\left\{\begin{array}{llll}
a^{2} & & & \\
& a^{q-1} & & \\
& & a^{q-1} & \\
& & & \bar{a}^{-2}
\end{array}\right) \mid \begin{array}{l}
s=a^{-q+3}+t c \\
u=a^{-q+3} c+a^{q-3} c+t c^{2}, \\
v=a^{q-3}+t c
\end{array} \\
& \left.\left(\begin{array}{cc}
s & t \\
u & v
\end{array}\right) \in S L_{2}(q), a \in G F\left(q^{2}\right)^{\times}\right\}
\end{aligned}
$$

Hence in particular $a^{q-3}+a^{-q+3} \in G F(q)$, so $a^{q-3}+a^{-q+3}=a^{1-3 q}+a^{-1+3 q}$. This implies that $a^{q-1}\left(a^{q}+a^{-1}\right)^{2}\left(a^{-(q+1)}+1\right)^{4}=0$. Hence $a^{q+1}=1$. Then, since $t=\left(a^{4}+a^{-4}\right) /(c+\bar{c}), \quad\left|\bar{x}^{I_{2} N_{2}}\right|=(q-1) q(q+1)\left(q^{2}-1\right) /(q+1)=(q-1) q\left(q^{2}-1\right)$. Now $(q+1)\left(q^{2}-1\right)+(q-1) q\left(q^{2}-1\right)=q^{4}-1=\left|\bar{A}_{2}^{\#}\right|$. Thus (2) holds. (3) is obtained by direct calculation.

(2.3) (1) $\mathscr{E}(P \bmod Z(P))=\left\{A_{1}, A_{2}\right\}$.

(2) $\operatorname{Jr}(\tilde{P})=A_{1}, \operatorname{Jr}\left(\tilde{P} \bmod A_{1}\right)=P$ and $\operatorname{Jr}(P \bmod Z(P))=A_{2}$.

(3) $\left|C_{A_{1}}(x)\right| \leq q^{2}$ for $x \in P-A_{1}$.

(4) $\left|C_{A_{1}}(x)\right| \leq q^{3}$ for $x \in \tilde{P}-P$.

(5) If $B$ is a maximal abelian subgroup of $P$ different from $A_{1}$, then $|B| \leq q^{3}$.

Proof. Set $\bar{P}=P / Z(P)$. Then $\bar{P}=\bar{A}_{1} \bar{A}_{2}$, where $\bar{A}_{1}$ and $\bar{A}_{2}$ are elementary abelian, and $C_{\bar{P}}(\bar{x})=\bar{A}_{1}$ for any $\bar{x} \in \bar{A}_{1}-\bar{A}_{2}$. This implies (1). For (5) assume that $B<A_{2}$. Set $(a, c, d)=\left(\begin{array}{llll}1 & & & \\ a & 1 & & \\ c & & 1 & \\ d & \bar{c} & \bar{a} & 1\end{array}\right)$. Let $x=\left(x_{1}, x_{2}, x_{3}\right) \in A_{2}-Z(P)$. Then $C_{A_{2}}(x)=\left\{(a, c, d) \mid a \bar{x}_{2}+\bar{a} x_{2}=c \bar{x}_{1}+\bar{c} x_{1}\right\}$. Hence $\left|C_{A_{2}}(x)\right|=q^{4}$. Moreover we have $C_{A_{2}}(x)=C_{A_{2}}\left(x^{\prime}\right)$ for any element $x^{\prime}=\left(k x_{1}, k x_{2}, x_{3}^{\prime}\right)$, where $k \in G F(q)$. Suppose that $y=\left(y_{1}, y_{2}, y_{3}\right) \in C_{A_{2}}(x)-Z(P)$ and that $y \neq\left(k x_{1}, k x_{2}, x_{3}^{\prime}\right)$ for any $k \in G F(q)$. Since $\left|C_{A_{2}}(x)\right|=q^{4}$ and $\left|\left\langle\left(k x_{1}, k x_{2}, x_{3}^{\prime}\right), Z(P) \mid k \in G F(q)\right\rangle\right|=q^{2}$, such $y$ really exists. 
Then $C_{A_{2}}(x, y)=\left\{(a, c, d) \mid a \bar{x}_{2}+\bar{a} x_{2}=c \bar{x}_{1}+\bar{c} x_{1}, \quad a \bar{y}_{2}+\bar{a} y_{2}=c \bar{y}_{1}+\bar{c} y_{1}\right\} . \quad$ Hence $\left|C_{A_{2}}(x, y)\right| \leq q^{3}$. Suppose that $x \in P-\left(A_{1} \cup A_{2}\right)$. Then it is easily obtained that $\left|C_{P}(x)\right|=q^{3}$. Thus (5) holds by (1). (3) and (4) follow from direct calculation. Now (3), (4) and (5) give that $\operatorname{Jr}(\tilde{P})=A_{1}$, since $q \geq 4$. By (1) we have $\operatorname{Jr}(P \bmod Z(P))=A_{2}$. We get $\operatorname{Jr}\left(\tilde{P} \bmod A_{1}\right)=P$ easily.

The following is an immediate consequence of (2.2) and (2.3.2).

(2.4) L has just two conjugacy classes of involutions which are represented by $z_{1}$ and $z_{2}$.

(2.5) (1) $C_{A}\left(I_{1}\right)=\left(I_{1} \times N_{1}\right)\langle u\rangle$ if $\left.q\right\rangle 4$, and $C_{A}\left(I_{1}\right)=\left(I_{1} \times N_{1}\right)\langle$ fr $\rangle$ if $q=4$. $C_{A}\left(I_{2}\right)=I_{2} N_{2}$.

(2) $C_{A}\left(A_{1}\right)=C_{A}\left(Z_{2}(P)\right)=A_{1}, C_{A}(Z(P))=A_{2} \cdot\left(I_{2}^{q-1} \times N_{2}\right)\langle u\rangle$.

(3) $C_{A}\left(A_{1} N_{1} / A_{1}\right)=A_{1} I_{1}, C_{A}\left(A_{2} N_{2} / A_{2}\right)=A_{2} I_{2}\langle u\rangle$.

(4) $C_{A}\left(A_{2} I_{2}^{q-1} / A_{2}\right)=C_{L}\left(A_{2} I_{2}^{q-1} / A_{2}\right)$.

(5) $C_{A}\left(P / A_{1}\right)=P I_{1}, C_{A}\left(A_{2} / Z(P)\right)=A_{2}$.

Proof. (1) is obtained by direct calculation. Similarly we have $C_{L}\left(A_{1}\right)=C_{L}\left(Z_{2}(P)\right)=A_{1}$. Hence by the Frattini argument $C_{A}\left(Z_{2}(P)\right)=A_{1}\left(N_{A}\left(A_{1}\right)\right.$ $\left.\cap C\left(Z_{2}(P)\right)\right)=A_{1}\left(\left(I_{1} N_{1}\langle f\rangle \cap C\left(Z_{2}(P)\right)\right)=A_{1}\right.$. By (2.1.5) $C_{A}(Z(P))=A_{2} I_{2}^{q-1} N_{2}\left(N_{A}(P)\right.$ $\cap C(Z(P)))=A_{2} I_{2}^{q-1} N_{2}(I\langle f\rangle \cap C(Z(P)))=A_{2} I_{2}^{q-1} N_{2}\langle u\rangle$. Thus (2) holds. By (2.1.4) $C_{A}\left(A_{1} N_{1} / A_{1}\right)=A_{1}\left(I_{1} N_{1}\langle f\rangle \cap C\left(I_{1}\right)\right)=A_{1} I_{1}$. Similarly $C_{A}\left(A_{2} I_{2} / A_{2}\right)=A_{2} I_{2}\langle u\rangle$, and $C_{A}\left(A_{2} I_{2}^{q-1} / A_{2}\right)=A_{2}\left(I_{2} N_{2}\langle f\rangle \cap C\left(I_{2}^{q-1}\right)\right)=A_{2} I_{2} N_{2}$, since any nontrivial element of $\langle f\rangle$ does not centralize $q+1$ elements of $G F\left(q^{2}\right)^{\times}$. This gives (4). (5) follows from (2.1.1) and (2.1.4).

The following is (19.8) (ii) of [4].

(2.6) Let $x \in I(A-L)$. Then $x$ is conjugate to $u$ or $u z_{1}$. Furthermore $C_{L}(u) \simeq P \operatorname{Sp}_{4}(q)$ and $C_{L}\left(u z_{1}\right)$ is isomorphic to the centralizer of a transvection in $\operatorname{PSp}_{4}(q)$.

(2.7) Any complement of $A_{1}$ in $A_{1} N_{1}$ is conjugate to $N_{1}$ in $A_{1} N_{1}$.

Proof. Let $M$ be a complement. Set $i(a)=\left(\begin{array}{llll}a & & & \\ & a^{-1} & & \\ & & \bar{a} & \\ & & \bar{a}^{-1}\end{array}\right) \in L$ and set $I^{\prime}=\left\{i(a) \mid a \in G F\left(q^{2}\right)^{\times}\right\}$. Then $N_{A_{1} N_{1}}(P)=P I^{\prime}$. So we may assume that $I^{\prime} \leq M$ by Schur-Zassenhauss' theorem. Set $\overline{A_{1} N_{1}}=A_{1} N_{1} / A_{1}$. Since $\bar{I}^{\prime}\left\langle\bar{r}_{1}\right\rangle=$

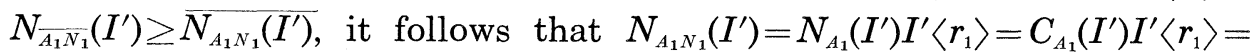
$I^{\prime}\left\langle r_{1}\right\rangle$. Therefore $N_{N_{1}}\left(I^{\prime}\right)=N_{M}\left(I^{\prime}\right)=I^{\prime}\left\langle r_{1}\right\rangle$. Then $M=\left\langle P \cap M, r_{1}\right\rangle$ by the structure of $M \simeq S L_{2}\left(q^{2}\right)$. Hence in order to prove the assertion we will 


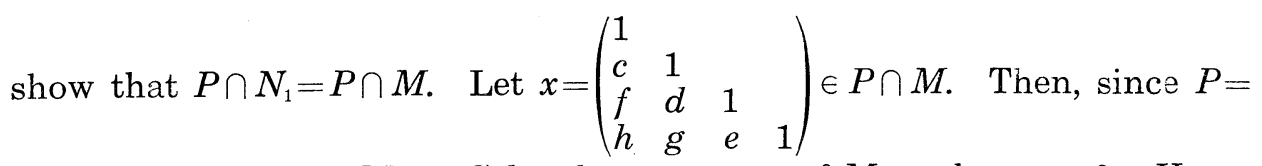
$A_{1} \cdot(P \cap M)$ and $(P \cap M)^{\sharp}=x^{I^{\prime}}$ by the structure of $M$, we have $c \neq 0$. Hence $(P \cap M)^{2}=1$, which yields $d=0$ and so $g=\bar{f}$. Since for any $a \in G F\left(q^{2}\right)^{\times}$there exists an element $b$ in $G F\left(q^{2}\right)^{\times}$such that $x x^{i(a)}=x^{i(b)}$, we have

$$
\left\{\begin{array}{l}
c+a^{2} c=b^{2} c \\
f+a \bar{a}^{-1} f=b \bar{b}^{-1} f \\
h+a \bar{a} h+\bar{a}^{2} \bar{c} f+a^{-1} \bar{a} c \bar{f}=b \bar{b} h .
\end{array}\right.
$$

Then provided that $f \neq 0$, we have $\bar{a}^{2}=a$ from the first two equalities. This yields $f=0$. Also we have $h=0$, which implies that $x \in P \cap N_{1}$. Thus $\left(P \cap N_{1}\right)^{\#}=x^{I^{\prime}}=(P \cap M)^{\sharp}$ and the proof is complete.

(2.8) Suppose that $x \in \tilde{P}-P$ and set $\bar{x}=x \tilde{P} / P . \quad$ If $|x|=|\bar{x}|$, them $m\left(C_{P}(x)\right)$ $=3 m$, where $m=2 n /|x|$.

Proof. Applying (1.4) to $P / A_{1}$, we may assume that $x=f^{i} a$ for some $a \in A_{1}$ and some integer $i$, where $f$ is the canonical field automorphism of $L$. Then $C_{A_{1}}(x)=C_{A_{1}}\left(f^{i}\right)$ and $m\left(C_{A_{1}}(x)\right)=3 m$. Moreover $m\left(C_{Z_{2}(P)}(x)\right)=2 m$. Since $P / A_{1} \simeq A_{2} / Z_{2}(P)$ as $N_{A}(P)$-modules, we have $\left|C_{P / A_{1}}(x)\right|=\left|C_{A_{2} / Z_{2}(P)}(x)\right|$, so $m\left(C_{A_{2} / Z_{2}(P)}(x)\right)=m$. Therefore $m\left(C_{A_{2}}(x)\right) \leq 3 m$. Now (2.3.1) implies that any elementary abelian subgroup of $P$ is contained in either $A_{1}$ or $A_{2}$. Thus we have $m\left(C_{P}(x)\right)=3 m$.

\section{§ 3. Fusion of the involution of $\mathrm{C}_{G}(\mathrm{~L})$}

In this section we begin the proof of the theorem stated in the introduction under the following hypothesis.

Hyротнеsis. Let $G$ be a finite group and let $L$ be a standard subgroup of $G$ such that $L / Z(L) \simeq U_{4}(q)$, where $q=2^{n} \geq 4$. Suppose that $O(G)=1$ and $L \bowtie G$. Furthermore suppose that $C(L)$ has a cyclic Sylow 2-subgroup.

It is known (cf. [15] [18]) that the Schur multiplier of $U_{4}(q), q \geq 4$, is trivial. So we may identify $L$ with the group of $4 \times 4$ matrices $x$ 's defined in the beginning of Section 2. The symbols used in Section 2 for various objects of $U_{4}(q)$ and Aut $\left(U_{4}(q)\right)$ will retain their meaning. Thus $P$ is a Sylow 2subgroup of $L$.

Let $t$ be an involution of $C(L)$ and set $C=C(t)$. Let $Q$ be a Sylow 2subgroup of $L C_{C}(L)$ containing $P$ and let $T$ be a Sylow 2-subgroup of $C$ containing $Q$. Finally, for $i=1,2$, we define $B_{i}=\langle t\rangle A_{i}$. 
(3.1) The following statements hold.

(1) $\operatorname{Jr}(T)=B_{1}$.

(2) Set $C_{T}\left(B_{1}\right)=\tilde{B}_{1}$. Then $t^{N\left(\tilde{B}_{1}\right)}=i^{G} \cap \tilde{B}_{1} \neq\{t\}$.

(3) $\tilde{B}_{1}=B_{1}$ and $\langle t\rangle \in S y l_{2}(C(L))$.

(4) $t^{G} \cap L=\phi$.

(5) $Q=\operatorname{Jr}\left(T \bmod B_{1}\right)$ and $B_{2}=\operatorname{Jr}(Q \bmod Z(Q))$.

(6) $t^{N\left(B_{1}\right)}=t A_{1}$.

Proof. Let $T_{0}=C_{T}(L)$. Then $Q=T_{0} \times P$ and $\operatorname{Jr}(Q)=B_{1}$ by (2.3.2). If $B$ is an abelian subgroup of $T$ of rank at least $4 n+1$, then $m\left(B T_{0} / T_{0}\right) \geq 4 n$, since $T_{0}$ is assumed to be cyclic by the hypothesis. So again by (2.3.2) $B \leq Q$. This proves (1). If $t^{G} \cap C=\{t\}$, then $t \in Z(G)$ by [9], since we are assuming $O(G)=1$. But then $C=G$ and $L \triangleleft G$, contrary to our hypothesis. So we may take $t^{g} \in C-L C(L)$. Then by (2.6) $t^{g h}=u, u z_{1}$, $t u$ or $t u z_{1}$ for some $h \in C$. Let $x=\left(\begin{array}{llll}1 & & & \\ a & 1 & & \\ c & (c+\bar{c}) / a & 1 & \\ d & c & a & 1\end{array}\right) \in L$. Then $t^{g h x}=t^{g h}\left(\begin{array}{cccc}1 & & & \\ 0 & 1 & & \\ c+\bar{c} & 0 & 1 & \\ 0 & c+\bar{c} & 0 & 1\end{array}\right)$. Since $L^{g h} \geq C_{L}\left(t^{g h}\right)^{(\infty)}$, we have $\left(\begin{array}{cccc}1 & & & \\ 0 & 1 & & \\ c+\bar{c} & 0 & 1 & \\ 0 & c+\bar{c} & 0 & 1\end{array}\right) \in L^{g h}$. Hence $t^{g h x} \in L^{g h} C\left(L^{g h}\right)$ $-\left\{t^{g h}\right\}$. So we may assume that $t^{g} \in L C(L)$. Then by (2.4) $t^{g k}=z_{1}, z_{2}, z_{1} t$ or $z_{2} t$ for some $k \in C$. Thus $t^{G} \cap B_{1} \neq\{t\}$. Let $s \in t^{G} \cap B_{1}-\{t\}$ and set $s^{g^{\prime}}=t$. Since $T \in S y l_{2}(C)$, we can choose $g^{\prime}$ such that $C_{T}(s)^{g^{\prime}} \leq T$. In particular $\tilde{B}_{1}^{g^{\prime}}<T$. Since $\tilde{B}_{1}=T_{0} \times A_{1} \geq B_{1}, \tilde{B}_{1}$ is weakly closed in $T$. Hence $\tilde{B}_{1}^{g^{\prime}}=\tilde{B}_{1}$ and $g^{\prime} \in N\left(\tilde{B}_{1}\right)$. Thus (2) holds. If $\left|T_{0}\right| \neq 2$, then $\langle t\rangle$ is a characteristic subgroup of $\tilde{B}_{1}$. But this contradicts (2). So (3) holds. Now (4) follows from (2.2.3), and (5) follows from (3) and (2.3.2).

By (2), (4) and (2.2.1) we have $t^{N\left(B_{1}\right)} \subseteq t A_{1}$, and $\left|t^{N\left(B_{1}\right)}\right|=q^{3}-q^{2}+q, q^{4}-q^{3}$ $+q^{2}-q+1$ or $q^{4}$. Set $\Omega=t^{N\left(B_{1}\right)}$. If either of the former two cases holds, then $N\left(B_{1}\right)^{\Omega}$ is doubly transitive and the one-point-stabilizer $N_{C}\left(B_{1}\right)^{\varrho}$ has a cyclic normal subgroup $I_{1}^{2}$. Notice that $N_{C}\left(B_{1}\right)^{\Omega}$ is not solvable. Hence by theorem 3 of [1] together with (1.2) neither of these cases hold. Thus $t^{N\left(B_{1}\right)}$ $=t A_{1}$.

(3.2) (1) $\quad N(Z(Q))=O_{2}(N(Z(Q))) N_{C}(Z(Q))$ and $\left|O_{2}(N(Z(Q)))\right|=2 q^{6}$.

(2) $N(Q)=O_{2}(N(Z(Q))) N_{C}(Q)$.

(3) $\quad N\left(B_{2}\right)=N(Z(Q))$.

Proof. Set $\Omega=t^{G} \cap Z(Q)$. Then $\Omega=t Z(P)$ by (3.1.4) and (3.1.6). (3.1.6) also implies that $T$ is not a Sylow 2-subgroup of $G$. Hence by (3.1.5) $t^{N(Z(Q))}$ $\neq\{t\}$. By (2.1.3) $N(Z(Q))^{\Omega}$ is doubly transitive and the one-point-stabilizer 
$N_{C}(Z(Q))^{\Omega}$ has a cyclic normal subgroup $I_{2}^{\Omega}$. Thus by (1.1) $N(Z(Q))^{\Omega}$ has a regular normal subgroup. Let $M$ be the preimage of the regular normal subgroup and let $X$ be a Sylow 2-subgroup of $M$. Then $M^{\Omega}=X^{\Omega}$, so $\left[X^{\Omega}, I_{2}^{\Omega}\right]$ $=X^{\Omega}$. Now $C(Z(Q))=B_{2} \cdot\left(I_{2}^{q-1} \times N_{2}\right) \cdot O(C)\left\langle u_{0}\right\rangle$ by $(2.5 .2)$, where $u_{0}=1$ or $u$ according as $Q=T$ or not. Since $C\left(I_{2}^{q-1} N_{2} O(C)\right) \geq I_{2}$, we have $\left[X, I_{2}\right] \leq$ $C\left(B_{2} I_{2}^{q-1} N_{2} O(C) / B_{2}\right)$ by three subgroup lemma. Let $X_{1} \in S y l_{2}\left(\left[X, I_{2}\right] B_{2}\right)$. Since $u$ acts on $I_{2}^{q-1}$ nontrivially, it follows that $u \notin\left[X, I_{2}\right] B_{2}$. Thus we have $M=$ $X_{1} \cdot\left(I_{2}^{q-1} \times N_{2}\right) O(C)\left\langle u_{0}\right\rangle$ and (1) holds.

Since $Q$ is characteristic in $T$ by (3.1.5), $N(Q)^{\Omega}$ is transitive as well as $N(Z(Q))^{2}$. So $\left|N(Q): N_{C}(Q)\right|=\left|N(Z(Q)): N_{C}(Z(Q))\right|=q$. Since $N(Q) \leq N(Z(Q))$, (2) follows. Since $Z\left(B_{2}\right)=Z(Q)$, we have (3) similarly.

(3.3) There exists a 2-subgroup $E$ of $C(P)$ of order $q^{2}$ such that $O_{2}(N(Z(Q)))$ $=E B_{2}, E \cap B_{2}=Z(P)$ and $E / Z(P) \simeq Z(P)$ as $N_{C}(Z(Q))$-modules.

Proof. Set $M=O_{2}(N(Z(Q)))$. Then $Z(M) \cap Z(P) \neq 1$, since $Z(Q) \triangleleft M$ and $t^{M}=Z(Q)-Z(P)$ by (3.2). Hence $Z(P) \leq Z(M)$ by (2.1.3). Now $M \cap C$ $=B_{2}$ and $Z\left(B_{2}\right)=Z(Q)$, so $Z(M) \leq Z(Q)$. Thus $Z(P)=Z(M)$ and $t \in Z_{2}(M)$. Then the map [,$t]$ from $M$ to $Z(P)$ is an $N_{C}(Z(P)$ )-homomorphism. Clearly the kernel is $B_{2}$ and the image is $Z(P)$. Hence $M=B_{2} C_{M}\left(I_{2}^{r_{1}}\right), B_{2} \cap C_{M}\left(I_{2}^{r_{1}}\right)$ $=Z(Q)$ and $C_{M}\left(I_{2}^{r_{1}}\right) / Z(Q) \simeq Z(P)$ as $I$-modules. Set $E=\left(C_{M}\left(I_{2}^{r_{1}}\right) I\right)^{\prime}$ and we will show that $E$ has the desired property.

$Z(P) \leq C_{M}\left(I_{2}^{r_{1}}\right)^{2} \leq Z(Q)$ by (2.1.3). If the second equality holds, then $t$ is a square involution in $C_{M}\left(I_{2}^{r_{1}}\right)$, since $t^{M}=Z(Q)-Z(P)$. But this contradicts the fact that $C_{M}(t)=B_{2}$. Hence $C_{M}\left(I_{2}^{r_{1}}\right) / Z(P)$ is elementary, $t \notin E$ and $C_{M}\left(I_{2}^{r_{1}}\right)$ $=E\langle t\rangle$. Then $E$ is of order $q^{2}$. Moreover $O_{2}(N(Z(Q)))=E B_{2}$ and $E \cap B_{2}=$ $Z(P)$. By (2.1.3) and (3.2.2) $Z_{2}(Q) \leq Z(M \bmod Z(Q))$. So $E Z(Q) \triangleleft E Z_{2}(Q)$. Since $Z_{2}(P)=Z(P)\left[Z_{2}(P), I_{2}^{r_{1}}\right]$, we have $\left[E Z(Q), Z_{2}(P)\right]=1$ by $(1.6)$. By definition $E$ is $r_{2}$-invariant. Hence $\left[E Z(Q), A_{2}\right]=1$, since $A_{2}=Z_{2}(P) Z_{2}(P)^{r_{2}}$. Then $E Z(Q)=C_{M}\left(A_{2}\right) \cap N(Z(Q))$ and $E=\left[E Z(Q), I_{2}\right]=\left[E Z(Q), M I_{2}\right] \triangleleft N(Z(Q))$. Hence $E / Z(P) \simeq Z(P)$ as $N_{C}(Z(Q))$-modules. Moreover by (1.6) we have $M Q=E Z(Q)$ $*\left[M Q, I_{2}^{r_{1}}\right]$. Since $P=\left[P, I_{2}^{r_{1}}\right]$ by (2.1.2), $M Q=E Z(Q) * P$ and the proof is complete.

(3.4) Set $D_{1}=O_{2}\left(N\left(B_{1}\right)\right)$. Then $N\left(B_{1}\right)=D_{1} N_{C}\left(B_{1}\right)$ and $\left|D_{1}\right|=2 q^{8}$. Furthermore $Z\left(D_{1}\right)=A_{1}$ and $D_{1} / B_{1} \simeq A_{1}$ as $N_{C}\left(B_{1}\right)$-modules.

Proof. Set $t^{N\left(B_{1}\right)}=\Omega$. Then by (2.2.1) and (3.1.6) $N\left(B_{1}\right)^{\Omega}$ is primitive. Since $A_{1}=B_{1}-\Omega$, we have $A_{1} \triangleleft N\left(B_{1}\right)$. Hence $\left(C\left(A_{1}\right) \cap N\left(B_{1}\right)\right)^{\Omega}$ is a nontrivial normal subgroup of $N\left(B_{1}\right)^{\Omega}$, since it contains $E^{\Omega}$. Hence it is transitive. Since $C\left(A_{1}\right) \cap N_{C}\left(B_{1}\right)=B_{1} O(C)$ by (2.5.2), $\left(C\left(A_{1}\right) \cap N\left(B_{1}\right)\right)^{2}$ is a regular normal subgroup of $N\left(B_{1}\right)^{2}$. Thus $N\left(B_{1}\right)=\left(C\left(A_{1}\right) \cap N\left(B_{1}\right)\right) N_{C}\left(B_{1}\right)$ and $\left(C\left(A_{1}\right) \cap N\left(B_{1}\right)\right)^{2}$ 
$\simeq A_{1}$ as $N_{C}\left(B_{1}\right)$-modules by the $[, t]$-homomorphism. Hence $N_{1}$ acts on $\left(C\left(A_{1}\right) \cap N\left(B_{1}\right)\right)^{2}$ irreducibly by (2.2.1). Since $N_{1} \leq C\left(B_{1} O(C) / B_{1}\right)$, we obtain that $C\left(A_{1}\right) \cap N\left(B_{1}\right)$ is 2-closed just as in (3.2). Therefore $D_{1}=O_{2}\left(C\left(A_{1}\right) \cap N\left(B_{1}\right)\right)$, $t^{D_{1}}=t A_{1}$ and $\left|D_{1}\right|=2 q^{8}$. Now it is clear that $D_{1} / B_{1} \simeq\left(C\left(A_{1}\right) \cap N\left(B_{1}\right)\right)^{\Omega}$ as $N_{C}\left(B_{1}\right)$-modules, which implies the last assertion.

(3.5) Set $F_{1}=\left(D_{1} I_{1}\right)^{\prime}, P_{1}=F_{1} P, Q_{1}=F_{1} Q, T_{1}=F_{1} T$ and $F=E C_{F_{1}}\left(I_{1}^{r_{2}}\right)$. Then the following statements hold.

(1) $D_{1}=F_{1}\langle t\rangle, F_{1} \triangleleft N\left(B_{1}\right)$ and $F_{1} / A_{1} \simeq A_{1}$ as $N_{C}\left(B_{1}\right)$-modules.

(2) $Z\left(P_{1}\right)=E, Z_{2}\left(P_{1}\right)=F$ and $Z_{3}\left(P_{1}\right)=P_{1}$.

(3) $F_{1}$ is elementary or homocyclic.

(4) $T_{1} \in \operatorname{Syl}_{2}\left(N\left(B_{1}\right)\right), J\left(T_{1}\right)=F_{1}$ and $\operatorname{Jr}\left(T_{1} \bmod F_{1}\right)=Q_{1}$.

(5) $N_{P_{1}}(P)=E P, N_{P_{1}}(E P)=F P$ and $N_{P_{1}}(F P)=P_{1}$.

(6) $E=C_{P_{1}}\left(I_{2}^{r_{1}}\right), F=E \cdot C_{P_{1}}\left(I_{1}^{r_{2}}\right), F_{1}=F \cdot C_{P_{1}}\left(I_{2}\right), P_{1}=F_{1} \cdot C_{P_{1}}\left(I_{1}\right)$.

Proof. By (3.4) $C_{D_{1}}\left(I_{1}\right)=\langle t\rangle$. Hence in order to prove (1) it is enough to show that $t \notin F_{1}$. Since $D_{1}$ is not elementary, $D_{1}^{2} \neq 1$. So by (2.2.1) $A_{1} \leq$ $D_{1}^{2} \leq B_{1}$. Suppose $D_{1}^{2}=B_{1}$. Then $t$ has a square root in $D_{1}$, since $t^{D_{1}}=t A_{1}$. But this yields that $C_{D_{1}}(t) \neq B_{1}$, which is a contradiction. Thus $D_{1} / A_{1}$ is elementary. So if we set $\overline{N\left(B_{1}\right)}=N\left(B_{1}\right) / A_{1}$, then $\bar{D}_{1}=\langle\bar{t}\rangle \times\left[\bar{D}_{1}, \bar{I}_{1}\right]$ and (1) follows.

By (2.1.2) $A_{1}=C_{P}\left(I_{2}^{q-1}\right) \times C_{P}\left(I_{1}^{r_{2}}\right)$. Hence $F_{1}=C_{F_{1}}\left(I_{2}^{q-1}\right) C_{F_{1}}\left(I_{1}^{r_{2}}\right)$ and $C_{F_{1}}\left(I_{2}^{q-1}\right) \cap C_{F_{1}}\left(I_{1}^{r_{2}}\right)=1$. Since $\left[I_{2}^{q-1}, I_{1}^{r_{2}}\right]=1$, by $(1 I)$ of [12] we have $F_{1}=$ $C_{F_{1}}\left(I_{2}^{q-1}\right) \times C_{F_{1}}\left(I_{1}^{r_{2}}\right)$. Since $C_{P}\left(I_{2}^{q-1}\right)=C_{P}\left(I_{2}^{r_{1}}\right) \times C_{P}\left(I_{2}\right), C_{F_{1}}\left(I_{2}^{q-1}\right)=C_{F_{1}}\left(I_{2}^{r_{1}}\right) \times C_{F_{1}}\left(I_{2}\right)$. sequently we have $F_{1}=C_{F_{1}}\left(I_{2}^{r_{1}}\right) \times C_{F_{1}}\left(I_{2}\right) \times C_{F_{1}}\left(I_{1}^{r_{2}}\right)$ and in particular $E=$ $C_{F_{1}}\left(I_{2}^{r_{1}}\right) \triangleleft F_{1}$. Now by (2.1.5) and (2.2.3) $Z(P) \cap Z(P)^{x}=1$ for $x \in N_{C}\left(A_{1}\right)$ $N(Z(P))$, so it follows that $\left[E, E^{x}\right]=1$. By the structure of $U_{4}(q)$ it is easily seen that $A_{1}=\left\langle Z(P)^{x} \mid x \in N_{C}\left(A_{1}\right)-N(Z(P))\right\rangle$. Hence $F_{1}=\left\langle E^{x}\right| x \in N_{C}\left(A_{1}\right)-$ $N(Z(P))\rangle$ and we have $E \leq Z\left(F_{1}\right)$, which implies that $F_{1}$ is abelian. Then, since $F_{1}^{2}=1$ or $A_{1}$ by (1), $F_{1}$ is elementary or homocyclic respectively.

$N_{P_{1}}(P)=N_{F_{1}}(P) P$. By $(1) N_{F_{1}}(P)=\left\{x \in F_{1} \mid[x, P] \leq A_{1}\right\}=\left\{x \in F_{1} \mid[x, t] \in Z(P)\right\}$ $=E A_{1}$. Hence $N_{P_{1}}(P)=E P$ and $C_{P_{1}}(P)=E$. Similarly $N_{F_{1}}(E P)=\left\{x \in F_{1} \mid[x, t]\right.$ $\left.\in Z_{2}(P)\right\}$. Since $\left|A_{1} E C_{F_{1}}\left(I_{1}^{r_{2}}\right)\right| A_{1}\left|=q^{3},\right|\left[E C_{F_{1}}\left(I_{1}^{r_{2}}\right), t\right] \mid=q^{3}$. Also $\left[E C_{F_{1}}\left(I_{1}^{r_{2}}\right), t\right]=$ $[E, t]\left[C_{F_{1}}\left(I_{1}^{r_{2}}\right), t\right] \leq Z(P) C_{A_{1}}\left(I_{1}^{r_{2}}\right)=Z_{2}(P)$ by $(2.1 .2)$, since $[E, t]=Z(P)$. Thus we have $N_{P_{1}}(E P)=F P$. Then $N_{P_{1}}(F P)=N_{F_{1}}(F P) P=F_{1} P=P_{1}$. Since $E=C_{P_{1}}\left(I_{2}^{r_{1}}\right)$ and $E=Z(E P)$, we have by (1.6) $E=Z(F P)$. Hence inductively we have $E=$ $Z\left(P_{1}\right)$. By (2.3.2) $J r(E P \bmod E)=E A_{2}$. Hence $N_{P_{1}}\left(E A_{2}\right) \leq N_{P_{1}}\left(E A_{2} A_{1}\right)=$ $N_{P_{1}}(E P)$, which gives $N_{P_{1}}\left(E A_{2}\right)=N_{P_{1}}(E P)$. Since $P_{1}=F_{1} P=F_{1} E A_{2}, F_{1} \cap E A_{2}$ $=E Z_{2}(P)$. Thus $F P / E Z_{2}(P)$ is abelian and so $F$ is normal in $P_{1}$. Then applying (1.6), we have $F / E \leq Z\left(P_{1} / E\right)$. Since $N_{P_{1}}(E P)=F P, Z\left(P_{1} / E\right) \leq F P / E$. Suppose $F / E<Z\left(P_{1} / E\right)$. Then $E Z_{2}(P) / E<E P / E \cap Z\left(P_{1} / E\right)$, which is absurd. 
Hence $F=Z_{2}\left(P_{1}\right)$. By $(1 I)$ of [12] $P_{1} / F=C_{P_{1} / F}\left(I_{1}\right) \times C_{P_{1} / F}\left(I_{2}\right)$. Note that $C_{P_{1} / F}\left(I_{1}\right)$ $\simeq C_{P / Z_{2}(P)}\left(I_{1}\right)$ and $C_{P_{1}}\left(I_{2}\right)<F_{1}$. So this gives $Z_{3}\left(P_{1}\right)=P_{1}$. Thus we get (2), (3) and (5). Also (6) is proved implicitly.

It remains to show (4). It is clear that $T_{1} \in \operatorname{Syl}_{2}\left(N\left(B_{1}\right)\right)$ by (3.4). (2.3.3) gives that $\left|C_{F_{1}}(x)\right| \leq q^{4}$ for $x \in P_{1}-F_{1}$. So $\left|C_{P_{1}}(x)\right| \leq q^{6}$ for $x \in P_{1}-F_{1}$. Hence $J\left(P_{1}\right)=F_{1}$. Let $B$ be an abelian subgroup of $T_{1}$ different from $F_{1}$. The above argument shows that $B \cap P_{1} \leq F_{1}$ or else $\left|B \cap P_{1}\right| \leq q^{6}$. If $B \nless P_{1}$ and $B \leq Q_{1}$, then $B \cap Z\left(P_{1}\right)=Z(P)$. Hence $\left|\left(B \cap P_{1}\right) Z(P)\right|=\left|B \cap P_{1}\right||Z(P)| /\left|B \cap Z\left(P_{1}\right)\right|$ and so $|B| \leq 2 n q^{6}<q^{8}$, since $q \geq 4$. If $B \nless Q_{1}$ and $B \cap P_{1} \nless \leq F_{1}$, then $|B| \leq 2 n q^{6}<q^{9}$ as above. Finally if $B \not Q_{1}$ and $B \cap P_{1} \leq F_{1}$, then by (2.3.4) $\left|C_{F_{1}}(x)\right| \leq q^{6}$ for $x \in T_{1}$ $-Q$ and this yields that $|B| \leq 2 n q^{6}$. Thus we have $J\left(T_{1}\right)=F_{1}$. Then (2.3.2) gives $\operatorname{Jr}\left(T_{1} \bmod F_{1}\right)=Q_{1}$, since $T_{1} / F_{1} \simeq T / A_{1}$.

\section{(3.6) $F_{1}$ is elementary.}

Proof. First we will show that $T_{1} \notin \operatorname{Syl}_{2}(G)$. By (3.3) $E B_{2}=E\langle t\rangle * A_{2}$. Hence $J\left(E B_{2}\right)=E A_{2}$, since $q \geq 4$. Then $E=Z\left(J\left(E B_{2}\right)\right)$. Set $\overline{N\left(E B_{2}\right)}=N\left(E B_{2}\right) / E$ and set $\Omega=\overline{t A}_{2}$. Since $Q_{1} / B_{1} \simeq P_{1} / A_{1}, \quad N_{Q_{1}}(E Q)=F Q$ by (3.5.5). Since $J(E Q \bmod E)=E B_{2}$ by (2.3.2), we have $F \leq N_{Q_{1}}\left(E B_{2}\right)$. Suppose that $\bar{x}^{f}=\bar{x}$ for $\bar{x} \in \Omega$ and $f \in F$. Then by (3.5.2) $\bar{t}^{f}=\bar{t}$, which implies $f^{\Omega}=1^{\Omega}$. Since $F=$ $E \times C_{F}\left(I_{1}^{r_{2}}\right)$ and each of these direct factors is $t$-invariant, we have $C_{F}(\Omega)=$ $N_{F}(\langle t\rangle E)=E Z_{2}(P)$. Hence $F^{\Omega}$ is semiregular of order $q^{2}$. By (2.2.2) and (3.2) $N_{C}\left(E B_{2}\right)^{2}$ has three orbits on $\Omega$ whose lengths are $1,(q+1)\left(q^{2}-1\right)$ and $(q-1) q\left(q^{2}-1\right)$. Now the action of $F^{\Omega}$ yields that any orbit of $N\left(E B_{2}\right)^{\Omega}$ has length divisible by $q^{2}$. Hence the only possibility remains that $N\left(E B_{2}\right)^{2}$ is transitive. Then the index $\left|N\left(E B_{2}\right): N(Z(Q))\right|$ is divisible by $q^{4}$, since $N(Z(Q))^{\Omega}$ is contained in the stabilizer of $\bar{t}$. By (3.2.1) and (3.3) ET $E$ $\operatorname{Syl}_{2}(N(Z(Q)))$. Thus the order of a Sylow 2-subgroup of $N\left(E B_{2}\right)$ is not less than $q^{5}|T|$, while $\left|T_{1}\right|=q^{4}|T|$. Therefore we have $T_{1} \notin \operatorname{Syl}_{2}(G)$.

Suppose that $\mathrm{F}$ is homocyclic. Then $F_{1}^{2}=A_{1}$ and $D_{1}=C_{T_{1}}\left(A_{1}\right)$ char $T_{1}$ by (2.5.2) and (3.5.4). If $t x t x=1$ for some $x \in F_{1}-A_{1}$, then $t x t x=1$ for any $x \in F_{1}$ $-A_{1}$, since $F_{1}=\left\langle x^{N^{(}\left(_{1}\right)}\right\rangle$ by (2.2.1). Hence if $t x t x \neq 1$ for some $x \in F_{1}-A_{1}$, then $t x t x \neq 1$ for any $x \in F_{1}-A_{1}$. In this case we get $\operatorname{Jr}\left(D_{1}\right)=B_{1}$. Then $B_{1}$ char $D_{1}$ char $T_{1}$, which is a contradiction, since $T_{1} \in \operatorname{Syl}_{2}\left(N\left(B_{1}\right)\right)$ and $T_{1} \notin$ $\operatorname{Syl}_{2}(G)$. Therefore we have $t x t x=1$ for any $x \in F_{1}-A_{1}$. Now the first paragraph shows that $N\left(E B_{2}\right)$ is transitive on $\overline{t A_{2}}$. So $t$ is $N\left(E B_{2}\right)$-conjugate to txa for some $x \in E$ and $a \in A_{2}-I\left(A_{2}\right)$. But $t x a t x a=t x t x a^{2}=a^{2} \neq 1$, a contradiction. Thus by (3.5.3) $F_{1}$ is elementary.

(3.7) Set $O_{2}(N(Q))=S$. Then $N\left(Q_{1}\right)=S N_{C}(Q)$ and $S / Q_{1} \simeq P / A_{1}$ as $N_{C}(Q)$ modules. Furthermore set $R=(S I)^{\prime}$. Then $S=R\langle t\rangle$. 
Proof. Set $\Omega=\left(Q_{1} / F_{1}\right)-\left(P_{1} / F_{1}\right)$. Since $T_{1} \notin \operatorname{Syl}_{2}(G)$, we can apply (1.1) to obtain that $N\left(Q_{1}\right)^{\Omega}$ has a regular normal subgroup. By (3.6) $\mathscr{E}\left(D_{1}\right)=$ $\left\{B_{1}, F_{1}\right\}$. So $N\left(D_{1}\right)=N\left(B_{1}\right)=D_{1} N_{C}\left(A_{1}\right)$. Then, since $Q_{1}=D_{1} Q$, we have $N\left(D_{1}\right)$ $\cap N\left(Q_{1}\right)=Q_{1} N_{C}(Q)$. Thus $C(\Omega)=C\left(Q_{1} / F_{1}\right)=Q_{1} C_{C}\left(Q / A_{1}\right)=Q_{1} I_{1} O(C)$ by (2.5.5). Therefore as in (3.2) we can obtain that the preimage of the regular normal subgroup is 2-closed and that $N\left(Q_{1}\right)=S N_{C}(Q)$. By the [, $t$-homomorphism we have $S / Q_{1} \simeq P_{1} / F_{1}$. Hence $S / Q_{1} \simeq P / A_{1}$ as $N_{C}(Q)$-modules. Since $N\left(D_{1}\right)=$ $N\left(B_{1}\right)$, the last assertion is obtained as in (3.5.1).

(3.8) Set $D_{2}=C_{S}\left(E A_{2} / E\right) \cap N\left(E B_{2}\right)$. Then $D_{2}=O_{2}\left(N\left(E B_{2}\right)\right), \quad N\left(E B_{2}\right)=$ $D_{2} N_{C}\left(B_{2}\right)$ and $D_{2} / E B_{2} \simeq A_{2} / Z(P)$ as $N_{C}\left(B_{2}\right)$-modules.

Proof. By (3.7) $R \cap Q_{1}=P_{1}$, so $E, F \triangleleft R$. Hence $F C_{R}\left(I_{1}\right) / E=\left(E C_{R}\left(I_{1}^{r_{2}}\right) / E\right)$ $\times\left(E C_{R}\left(I_{1}\right) / E\right)$ by (1I) of [12]. Then $E A_{2} \leq Z\left(F C_{R}\left(I_{1}\right) \bmod E\right)$, since $F C_{R}\left(I_{1}\right) \cap$ $P_{1}=F C_{P_{1}}\left(I_{1}\right)=F C_{P}\left(I_{1}\right)>E A_{2}$ and since $I$ acts on $C_{P}\left(I_{1}\right)^{*}$ transitively by (2.1.3). Hence by (3.5) and (3.7) $F C_{S}\left(I_{1}\right)=C_{S}\left(E A_{2} / E\right)$. Now $E B_{2} / E=\left(E Z_{2}(P) / E\right) \times$ $\left(E C_{Q_{1}}\left(I_{1}\right) / E\right)$. Moreover $E Z_{2}(P) \cap E C_{R}\left(I_{1}^{r_{2}}\right)=F$ and $R$ normalizes $Q_{1}$. These facts yield that $E B_{2} \triangleleft F C_{S}\left(I_{1}\right)$. Notice that $\left|C_{S}\left(I_{1}\right) F: E B_{2}\right|=q^{4}$. By (3.6) $E$ is elementary. Hence $\mathscr{E}(\langle t\rangle E)=\{Z(Q), E\}$ and so $N(\langle t\rangle E)=N(Z(Q))=E N_{C}\left(B_{2}\right)$ by (3.3). Then $E N_{C}\left(B_{2}\right) \cap C\left(E A_{2} / E\right)=E B_{2} O(C)$ by (2.5.5). Therefore if we set $\Omega$ as in the proof of (3.6), then it follows that $\left(C_{S}\left(I_{1}\right) F\right)^{\Omega}$ is a regular normal subgroup of $N\left(E B_{2}\right)^{\Omega}$. Since $I_{2} \times N_{2}<C(O(C))$ and it acts on $\left(C_{S}\left(I_{1}\right) F\right)^{\Omega}$ irreducibly by (2.2.2), it is obtained that the preimage of $\left(C_{S}\left(I_{1}\right) F\right)^{\Omega}$ is 2-closed as in the proof of (3.2.1). Hence $D_{2}=C_{S}\left(I_{1}\right) F$ and $N\left(E B_{2}\right)=D_{2} N_{C}\left(B_{2}\right)$. Then by the $[, t]$-homomorphism we have $D_{2} / E B_{2} \simeq F A_{2} / E$ as $N_{C}\left(B_{2}\right)$-modules. Since $E A_{2} / E \simeq A_{2} / Z(P)$, the last assertion follows.

(3.9) (1) $Z(R)=E, Z_{2}(R)=F, Z_{3}(R)=R, F_{1}$ char $R$.

(2) Set $F_{2}=D_{2} \cap R$. Then $F_{2} \triangleleft N\left(E B_{2}\right), F_{2} / E$ is elementary and $F_{2} / E A_{2}$ $\simeq E A_{2} / E \simeq A_{2} / Z(P)$ as $N_{C}\left(B_{2}\right)$-modules.

(3) $E=C_{R}\left(I_{2}^{r_{1}}\right), F=E \cdot C_{R}\left(I_{1}^{r_{2}}\right), F_{i}=F \cdot C_{R}\left(I_{3-i}\right), R=F_{i} \cdot C_{R}\left(I_{i}\right), i=1,2$.

Proof. By (3.5.6) and (3.7) $E=C_{R}\left(I_{2}^{r_{1}}\right)$ and $F=E \cdot C_{R}\left(I_{1}^{r_{1}}\right)$. By (1I) of [12] $R / F=\left(F C_{R}\left(I_{1}\right) / F\right) \times\left(F C_{R}\left(I_{2}\right) / F\right)$. Hence $F_{i}=F \cdot C_{R}\left(I_{3-i}\right)$ and $R=F_{i} \cdot C_{R}\left(I_{i}\right)$, $i=1,2$. Thus (3) holds.

By (1.6) $E \leq Z(R)$. Suppose that $E<Z(R)$. Then by (3) $Z(R) \not P_{1}$ and $\left[Z(R), I_{1}\right] \leq P_{1}$. Hence $Z(R) \cap C\left(I_{1}\right) \neq 1$. Since $Z(R) \cap C\left(I_{1}\right)$ is $t$-invariant, we have $Z(R) \cap C\left(I_{1}\right) \cap C \neq 1$, which contradicts (2.1.2). Similarly we have $Z_{2}(R)$ $=F$. By (3.7) and (3.8) $F_{2}=\left(D_{2} I_{2}\right)^{\prime}$, so $F_{2} \triangleleft N\left(E B_{2}\right)$, since $N\left(E B_{2}\right)=D_{2} N_{C}\left(B_{2}\right)$ $\triangleleft D_{2} I_{2}$. Then $\left(F_{2} / E\right)^{2}=\left(C_{R}\left(I_{1}\right) E / E\right)^{2} \times(F / E)^{2}=\left(C_{R}\left(I_{1}\right) E / E\right)^{2}<E A_{2} / E$. Since $I_{2} \times N_{2}$ acts on $E A_{2} / E$ irreducibly, we have $F_{2}^{2} \leq E$. Thus (2) holds and at the same time we have $Z_{3}(R)=R$. By (3.6) $C_{R}(F) \geq F_{1}$. If $C_{R}(F)>F_{1}$, then by (3) 
$C_{R}(F) \cap C_{R}\left(I_{1}\right) \neq 1$. Hence $C \cap C_{R}(F) \cap C_{R}\left(I_{1}\right) \neq 1$. This yields that $C_{P}\left(Z_{2}(P)\right)$ $\cap C_{P}\left(I_{1}\right) \neq 1$, which contradicts (2.1.2) and (2.5.2). Thus $C_{R}(F)=F_{1}$ and (1) holds.

(3.10) $\mathscr{E}\left(D_{1}\right)=\left\{F_{1}, B_{1}\right\}$ and $\mathscr{E}\left(D_{2} \bmod Z_{2}\left(D_{2}\right)\right)=\left\{F_{2}, E B_{2}\right\} . \quad$ In particular $N\left(D_{1}\right)=N\left(B_{1}\right)$ and $N\left(D_{2}\right)=N\left(E B_{2}\right)$.

Proof. By (3.6) $\mathscr{E}\left(D_{1}\right)=\left\{F_{1}, B_{1}\right\} . \quad Z\left(D_{2}\right) \leq Z\left(C_{D_{2}}(t)\right)=Z\left(B_{2}\right)=Z(Q)$. Since $t \notin Z\left(D_{2}\right), Z(P)=Z\left(D_{2}\right)$ by (2.1.3). Then $E \leq Z_{2}\left(D_{2}\right)$, since $E \triangleleft D_{2}$ and since $I$ acts on $E / Z(P)$ irreducibly by (3.3). If $E<Z_{2}\left(D_{2}\right)$, then $E A_{2}=Z_{2}\left(D_{2}\right)$, since by (3.8) $N_{C}\left(B_{2}\right)$ acts irreducibly on $E A_{2} / E=Z\left(D_{2} / E\right)$. This yields that $A_{2} F / Z_{2}(P)$ $\simeq A_{1} A_{2} F / A_{1} \simeq F P / A_{1}$, which is not abelian by (3.5.5). This is a contradiction. Thus $E=Z_{2}\left(D_{2}\right)$ and by (3.9.2) we have $\mathscr{E}\left(D_{2} \bmod Z_{2}\left(D_{2}\right)\right)=\left\{F_{2}, E B_{2}\right\}$.

(3.11) (1) $E^{r_{1}} \cap F=1$.

(2) $C_{R}\left(I_{1}^{r_{2}}\right)^{r_{2}} \cap F=1$.

Proof. By (3.9.3) $E^{r_{1}}=C_{R}\left(I_{2}^{r_{1}}\right)^{r_{1}}=C_{F_{1}}\left(I_{2}^{r_{1}}\right)^{r_{1}}=C_{F_{1}}\left(I_{2}\right)$, since $F_{1}$ is $r_{1}{ }^{-}$ invariant. Then (1) follows from (3.9.3) again. We can obtain (2) similarly.

(3.12) Set $M_{1}=\left\langle N_{1}^{N\left(F_{1}\right) \cap C\left(I_{1}\right)}\right\rangle$ and set $M_{2}=\left\langle N^{N\left(F_{2}\right) \cap C\left(I_{2}\right)}\right\rangle$. Then the following statements hold.

(1) $M_{1} \simeq S L_{2}\left(q^{2}\right) \times S L_{2}\left(q^{2}\right)$ or $S L_{2}\left(q^{4}\right)$.

(2) $\quad M_{2} \simeq S L_{2}(q) \times S L_{2}(q)$ or $S L_{2}\left(q^{2}\right)$.

Moreover in either case, $t$ interchanges the components of $M_{i}$ if the former holds, and $t$ acts on $M_{i}$ as a field automorphism if the latter holds.

Proof. By (2.5.1) and (3.5.1) $N_{1}$ is a standard subgroup of $N\left(F_{1}\right) \cap C\left(I_{1}\right)$ and $\langle t\rangle$ is a Sylow 2-subgroup of $N\left(F_{1}\right) \cap C\left(I_{1}\right) \cap C\left(N_{1}\right)$. By (3.9) $t$ is not a central involution in $N\left(F_{1}\right) \cap C\left(I_{1}\right)$ and $C_{R}\left(I_{1}\right)$ is elementary We also have $\left[C_{R}\left(I_{1}\right), I \cap N_{1}\right]=C_{R}\left(I_{1}\right)$. Hence we can appeal to the result of [16] to obtain (1). (2) is obtained similarly.

Definition. $\quad L_{1}=F_{1} \cdot M_{1}$ and $L_{2}=F_{2} \cdot M_{2}$.

(3.13) (1) $L_{1} \triangleleft N\left(F_{1}\right)$.

(2) $E=Z\left(L_{2}\right)$.

(3) $L_{1}$ and $L_{2} / E$ are perfect.

Proof. Set $\overline{N\left(F_{1}\right)}=N\left(F_{1}\right) / F_{1}$. By (2.5.3) and (3.10) $\bar{N}_{1}$ is a standard subgroup of $\overline{N\left(F_{1}\right)}$ and $\langle\bar{t}\rangle$ is a Sylow 2-subgroup of $C_{\overline{N\left(F_{1}\right)}}\left(\bar{N}_{1}\right)$. Then as in (3.12) we have $\left\langle\bar{N}_{1}^{\overline{N\left(F_{1}\right)}}\right\rangle \simeq S L_{2}\left(q^{2}\right) \times S L_{2}\left(q^{2}\right)$ or $S L_{2}\left(q^{4}\right)$. Since $\bar{M}_{1} \leq\left\langle\bar{N}_{1}^{N\left(F_{1}\right)}\right\rangle, L_{1}$ is the preimage of $\left\langle\bar{N}_{1}^{N\left(F_{1}\right)}\right\rangle$, so $L_{1} \triangleleft N\left(F_{1}\right)$

Now $E \leq Z\left(F_{2}\right)$. Suppose that $E<Z\left(F_{2}\right)$. Then by (3.9.2) $Z\left(F_{2}\right) A_{2}=F_{2}$. 
Since $Z\left(F_{2}\right) \cap A_{2}=Z(P)$ and $\left|F_{2}\right|=q^{10}$, it follows that $\left|Z\left(F_{2}\right)\right|=q^{6}$. By (3.9.3) $R=F_{1} F_{2}$ and $F_{1} \cap F_{2}=F$. Therefore $Z\left(F_{2}\right) \cap F=E$. Then considering the order, we have $Z\left(F_{2}\right) F=F_{2}$, a contradiction. Hence $E=Z\left(F_{2}\right)$. By (2.1.5) and (3.3) $N_{2}<C(E)$, since $N_{2}=O^{2^{\prime}}\left(N_{2}\right)$. So $C_{M_{2}}(E)$ is a nontrivial $t$-invariant normal subgroup of $M_{2}$. Then the structure of $M_{2}$ yields that $C_{M_{2}}(E)=M_{2}$. Thus $E=Z\left(L_{2}\right)$.

By (2.2.1) and (3.5.1) $F_{1} N_{1}$ is perfect. Hence $L_{1}=F_{1} M_{1}$ is perfect. Similarly $L_{2} / E$ is perfect by (2.2.2) and (3.9.2).

(3.14) (1) $R \in \operatorname{Syl}_{2}\left(L_{1}\right) \cap \operatorname{Syl}_{2}\left(L_{2}\right)$.

(2) $N_{L_{1}}(R)$ and $N_{L_{2}}(R)$ normalize each other.

(3) $\quad N_{L_{1}}(R) \cap N_{L_{2}}(R)=R$.

Proof. (1) is a direct consequence of (3.12). By (3.9.1) and (3.13.1) $N_{L_{2}}(R)$ normalizes $N_{L_{1}}(R)$. Set $J_{1}=N_{M_{1}}(R) \cap N_{M_{1}}\left(R^{r_{1}}\right)$. Then $N_{M_{1}}(R)=$ $\left(R \cap M_{1}\right) \cdot J_{1}$ and $I \cap M_{1}<J_{1}$. Since $J_{1} \simeq Z_{q^{2-1}} \times Z_{q^{2-1}}$ or $Z_{q^{4-1}}$ and since $I=I_{1} \times\left(I \cap N_{1}\right)$, we have $I_{1} \times J_{1}<C\left(I_{2}\right)$. By (3.9.3) $F_{2}=\left(R I_{2}\right)^{\prime}$. Hence $J_{1}<$ $N\left(F_{2}\right) \cap C\left(I_{2}\right)$. This proves (2).

By the choice of $J_{1}, r_{1}$ inverts the elements of $J_{1}$. Set $N_{M_{2}}(R)=\left(R \cap M_{2}\right)$ . $J_{2}$. Then $\left(J_{1} \cap R J_{2}\right)^{r_{1}}=J_{1} \cap R J_{2}$. By (3.13.2) $E \leq C\left(J_{1} \cap R J_{2}\right)$. By (3.11.1) $F_{1}=F \cdot E^{r_{1}}$. Therefore $J_{1} \cap R J_{2}$ acts on $F_{1} / F$ trivially. Since $F_{1} / F \simeq R / F_{2}$ and since any non-identity element of $J_{2}$ acts on $R / F_{2}$ nontrivially, we have $J_{1} \cap R J_{2}=1$. Since $R J_{1} \cap R J_{2}=R\left(J_{1} \cap R J_{2}\right)$, (3) is obtained.

$$
L_{1} / F_{1} \simeq S L_{2}\left(q^{2}\right) \times S L_{2}\left(q^{2}\right) .
$$

Proof. Assume that the assertion does not hold. Let $J_{1}$ be as in (3.14). Then $I \cap J_{1}=I \cap M_{1}=I \cap N_{1}$. Suppose that $L_{2} / F_{2} \simeq S L_{2}\left(q^{2}\right)$. Then $J_{2}$ $\simeq Z_{q^{2-1}}$. By (3.14) $J_{1} J_{2} R / R \simeq Z_{q^{4-1}} \times Z_{q^{2}-1}$. So we may take $J_{2}$ such that $J_{1} J_{2}$ $=J_{1} \times J_{2}$. Then $\left(J_{1} J_{2}\right)^{\left(q^{2}+1\right)(q-1)}=\left(J_{1}^{q^{2}+1} J_{2}\right)^{q-1}=\left(I \cap N_{1}\right)^{q-1} J_{2}^{q-1} \simeq Z_{q+1} \times Z_{q+1}$. Since $q=2^{n}, J_{1} J_{2} / C_{J_{1} J_{2}}\left(L_{1} / F_{1}\right)$ acts on $L_{1} / F_{1}$ as an inner automorphism. Therefore $C\left(L_{1} / F_{1}\right) \cap\left(I \cap N_{1}\right)^{q-1} J_{2}^{q-1} \neq 1$. By (2.1.2) and (3.3) $\left(I \cap N_{1}\right)^{q-1}<C(E)$. So by (3.11.1) $\left(I \cap N_{1}\right)^{q-1}<C\left(F_{1} / F\right)$, since $I$ and $N_{1}$ are $r_{1}$-invariant. Also by (3.13.2) $J_{2}^{q-1}<C(E)$ and $J_{2}^{q-1} \not C C\left(F_{1} / F\right)$, since $F_{1} / F \simeq R / F_{2}$. Consequently $F_{1}$ has a nontrivial $L_{1}$-invariant subspace $C_{F_{1}}\left(C\left(L_{1} / F_{1}\right)\right) \cap\left(I \cap N_{1}\right)^{q-1} J_{2}^{q-1}$. But this can not hold, since $L_{1}$ acts on $F_{1}$ irreducibly by Lemma $4 \mathrm{~B}$ of [8]. Suppose that $L_{2} / F_{2} \simeq S L_{2}(q) \times S L_{2}(q)$. Then $J_{2} \simeq Z_{q-1} \times Z_{q-1}$ and by $(3.14) C_{J_{2}}\left(L_{1} / F_{1}\right) \neq 1$. Then we can derive a contradiction in the same way as above.

Definition. Set $L_{1}=K_{1} K_{1}^{t}$ and $U=R \cap K_{1}$, where $K_{1} \triangleleft L_{1}$ and $K_{1} / F_{1} \simeq$ $S L_{2}\left(q^{2}\right)$.

(3.16) (1) $|Z(U)| \geq q^{4}$. 
(2) If $|Z(U)|=q^{4}$, then $Z(U)<F$.

Proor. Suppose that $|Z(U)|<q^{4}$. Then by Lemma $4 \mathrm{~B}$ of [8] $K_{1}^{t} \leq C(Z(U))$. By symmetry $K_{1} \leq C\left(Z\left(U^{t}\right)\right)$. Then $K_{1} K_{1}^{t} \leq C\left(Z(U) \cap Z\left(U^{t}\right)\right)$, which is a contradiction, since $Z(U) \cap Z\left(U^{t}\right)=E$ and $N_{1} \nless C(Z(P))$. Thus (1) holds.

Since $F=Z_{2}(R)$ and $E=Z(R)<Z(U)$ by (3.9.1), it follows that $E<Z(U)$ $\cap F$. By (3.9.3) $I / I_{1}^{r_{2}}$ acts on $F / E$ fixed-point-freely. Then, since $I=I_{1}^{r_{2}} \times I_{2}^{r_{2}}$, any $I_{2}^{r_{2}}$-orbit on $(F / E)^{*}$ is of length $q^{2}-1$. This yields that $(Z(U) \cap F) / E$ is of order at least $q^{2}$. Thus (2) holds.

For the following we refer the readers to (4I) of [12].

(3.17) Suppose $Z(U) \leq F$. Then the following statements hold.

(1) $F_{1}=Z(U) \times Z\left(U^{r_{1}}\right)$.

(2) $F=Z(U) Z\left(U^{t}\right)$.

(3) $K_{1}^{t}$ acts on $Z(U)$ and on $Z\left(U^{r_{1}}\right)$ as $S L_{2}\left(q^{2}\right)$ acts naturally on a 2dimensional vector space over $G F\left(q^{2}\right)$.

(4) If $R \neq R^{\prime} \in \operatorname{Syl}_{2}\left(L_{1}\right)$, then $Z(R) \cap Z\left(R^{\prime}\right)=1$.

(5) If $z \in E^{\sharp}$, then $z^{N\left(F_{1}\right)} \cap F=Z(U)^{\ddagger} \cup Z\left(U^{t}\right)^{\ddagger}$.

Proof. Suppose $Z(U) \cap Z\left(U^{r_{1}}\right) \neq 1$. Then, since $K_{1}^{t}$ normalizes $Z(U)$ $\cap Z\left(U^{r_{1}}\right)$, we have $Z\left(U^{t}\right) \cap Z(U) \cap Z\left(U^{r_{1}}\right) \neq 1$. This implies that $1 \neq E \cap Z\left(U^{r_{1}}\right)$ $\leq E \cap F^{r_{1}}$, which contradicts (3.11.1). Thus (3.16.1) gives (1). Since $Z(U) \cap$ $Z\left(U^{t}\right)=E$, (2) follows. In particular $Z(U) \neq Z\left(U^{t}\right)$. Hence $K_{1}^{t}$ acts on $Z(U)$ nontrivially. So we can appeal to $(1 K)$ of [12] to obtain (3). By the structure of $S L_{2}\left(q^{2}\right) \times S L_{2}\left(q^{2}\right), R^{\prime}$ is $R$-conjugate to one of $R^{r_{1}}, U U^{r_{1} t}$ and $U^{r_{1}} U^{t}$. Hence for the proof of (4) we may assume that $R^{\prime}$ is one of those. Then in any case we have $E \cap Z\left(U^{r_{1}}\right), E \cap Z\left(U^{r_{1} t}\right) \leq E \cap F^{r_{1}}=1$ by (3.11.1). Thus (4) holds. Since $F=Z_{2}(R)$, arguing as in (4), we see that it is enough for (5) to show that $Z\left(R^{\prime}\right) \cap F \subseteq Z(U) \cup Z\left(U^{t}\right)$ for $R^{\prime}$ mentioned in (4), and this obviously holds.

\section{§. Case for $L_{4}\left(q^{2}\right)$}

In this section we assume the following and we will obtain that $\left\langle L^{G}\right\rangle$ $\simeq L_{4}\left(q^{2}\right)$ under the hypothesis made at the beginning of Section 3 .

HYротнеSIS. $L_{2} / F_{2} \simeq S L_{2}\left(q^{2}\right)$.

(4.1) (1) $\mathscr{E}(R \bmod E)=\left\{F_{1}, F_{2}\right\}$.

(2) $Z(U)<F$.

Proof. If we show $I(R)=I\left(F_{1}\right) \cup I\left(F_{2}\right)$, then (3.6) and (3.9.2) will give (1). Suppose false and let $x \in I(R)-I\left(F_{1}\right)-I\left(F_{2}\right)$. Then by our hypothesis in this 
section $J_{2}$ acts on $\left(R / F_{2}\right)^{\#}$ transitively. Since $F_{1}$ char $R$ (see (3.9.1)), we have $x^{y} \notin I(R)-I\left(F_{1}\right)-I\left(F_{2}\right)$ for any $y \in J_{1}$. So we may take $x=f a_{1} \notin F_{1} \cup F_{2}$ for some $f \in F_{2}$ and $a_{1} \in A_{1}-Z_{2}(P)$. Then by (3.9.2) $[f, t] \in Z_{2}(P) E$. Hence $f \in F A_{2}$. Set $f=a_{2} f^{\prime}$ with $a_{2} \in A_{2}, f^{\prime} \in F$ and set $\bar{R}=R / E$. Then $\overline{1}=\left(\bar{a}_{2} \bar{f}^{\prime} \bar{a}_{1}\right)^{2}=\left(\bar{a}_{2} \bar{a}_{1}\right)^{2}$ by (3.9.1). But, since $E P / E \simeq P / Z(P)$, this yields that $a_{2} \in A_{1} \cap A_{2}$ and so $x \in F_{1}$, a contradiction. Thus (1) holds. Then (2) follows from (1), since $F=F_{1} \cap F_{2}$ $<U$.

$$
C_{J_{2} L_{1}}\left(z_{2}\right)=C_{L_{1}}\left(z_{2}\right) \text { and } C_{L_{1}}\left(z_{2}\right) / F_{1} \simeq S L_{2}\left(q^{2}\right)
$$

Proof. By (2.2.3) $S L_{2}(q) \simeq C_{N_{1}}\left(z_{2}\right) \leq C_{M_{1}}\left(z_{2}\right)$. Since $C_{M_{1}}\left(z_{2}\right)$ is $t$-invariant, $C_{M_{1}}\left(z_{2}\right) \simeq S L_{2}(q), S L_{2}(q) \times S L_{2}(q)$ or $S L_{2}\left(q^{2}\right)$ by the structure of $M_{1}$. If $C_{M M_{1}}\left(z_{2}\right)$ $\simeq S L_{2}(q)$, then $\left|z_{2}^{L_{1}}\right|=\left|M_{1}: C_{M M_{1}}\left(z_{2}\right)\right|>\left|F_{1}\right|$. Hence this can not occur. So in any case $C_{M_{1}}\left(z_{2}\right)$ is maximal among the subgroups of $M_{1}$ which are $t$-invariant.

Now by (3.14) $J_{2} L_{1} / F_{1} \simeq Z_{q^{2-1}} \times S L_{2}\left(q^{2}\right) \times S L_{2}\left(q^{2}\right)$. Set $\overline{J_{1} J_{2}}=J_{1} J_{2} R / R$ and set $\bar{J}=C_{\left(J_{1} \cap K_{1}\right) J_{2}}\left(U / F_{1}\right)$. Then $\bar{J} \simeq Z_{q^{2-1}}$. Let $\langle\bar{x}\rangle=\bar{J}$ and let $\langle\bar{y}\rangle=\bar{J}_{2}$. Then $\overline{x y^{i}} \in \overline{J_{1} \cap K_{1}}$ for some integer $i$. So $\bar{x}$ acts on $U^{t} / F_{1}$ as $\bar{y}^{-i}$ acts on it. Since $t$ acts on $L_{2} / F_{2}$ as a field automorphism, we have $\bar{y}^{t}=\bar{y}^{q}$. Hence $\bar{x}^{t}$ centralizes $U^{t} / F_{1}$ and acts on $U / F_{1}$ as $\bar{y}^{-q i}$ acts on it. Therefore $\bar{x} \bar{x}^{q t} \bar{y}^{i}$ centralizes $R / F_{1}$. Set $\Omega=E^{\#}$ and we will show $\left\langle x x^{q t} y^{i}\right\rangle^{\Omega} \simeq Z_{q^{2}}$. By (3.13.2) $\left(x x^{q t} y^{i}\right)^{\Omega}=$ $\left(\left(x y^{i}\right)\left(x^{q} y^{i q}\right)^{t}\right)^{\Omega}$. Since $\langle t\rangle N_{L_{1}}(R)$ normalizes $E$ and since $N_{L_{1}}(R)^{\Omega}$ is transitive, we can apply (1.3) to obtain $\left(\left(x y^{i}\right)^{t}\right)^{\Omega}=\left(\left(x y^{i}\right)^{q}\right)^{\Omega}$. Hence $\left\langle x x^{q t} y^{i}\right\rangle^{\Omega}=\left\langle x y^{i}\right\rangle^{\Omega}$. Then, since $\left(J_{1} \cap K_{1}\right)^{\Omega}$ is faithful by (3.17.3), we have $\left\langle x x^{q t} y^{i}\right\rangle^{\Omega} \simeq\left\langle\bar{x} \bar{y}^{i}\right\rangle$. So in order to see $\left\langle x x^{q t} y^{i}\right\rangle^{\Omega} \simeq Z_{q^{2-1}}$ it is enough to show that $\bar{J} \cap \bar{J}_{2}=\overline{1}$. Since $J_{2}$ is cyclic, $\bar{J} \cap \bar{J}_{2}$ is $t$-invariant. So $\bar{J} \cap \bar{J}_{2}<C\left(R / F_{1}\right)$. Hence if $R \cdot J_{0}$ denotes the preimage of $\bar{J} \cap \bar{J}_{2}$, then $J_{0}<C\left(L_{1} / F_{1}\right)$. By (3.13.2) $J_{0} F_{1}<C(E)$ and so by (3.17.3) and (4.1.2) we have $J_{0} F_{1}<C\left(F_{1}\right)$. In particular $J_{0} \leq C\left(R / F_{2}\right)$, since $F_{1} / F \simeq R / F_{2}$ by (3.9). This implies $J_{0}=1$ by the structure of $L_{2} / F_{2}$. Thus $\left\langle x x^{q t} y^{i}\right\rangle^{\Omega}=C_{J_{1} J_{2}}\left(R / F_{1}\right)^{\Omega} \simeq Z_{q^{2-1}}$. In other words $C_{J_{2} L_{1}}\left(L_{1} / F_{1}\right)^{\Omega} \simeq Z_{q^{2-1}}$.

By $(2.2 .1)\left\langle z_{2}^{N 1}\right\rangle=A_{1}$, and so $\left\langle z_{2}^{L_{1}}\right\rangle=\left\langle A_{1}^{L_{1}}\right\rangle=F_{1}$. Notice that $C_{J_{2} L_{1}}\left(L_{1} / F_{1}\right)$ acts on the set of the orbits of $L_{1}$ on $F_{1}$. Let $J^{\prime} F_{1}$ be the subgroup of $C_{J_{2} L_{1}}\left(L_{1} / F_{1}\right)$ which leaves the orbit $z_{2}^{L_{1}}$ invariant. Set $J^{\prime \prime}=C_{J^{\prime}}\left(z_{2}^{L_{1}}\right)$. Then $\left[J^{\prime \prime}, F_{1}\right]=1$, since $\left\langle z_{2}^{L_{1}}\right\rangle=F_{1}$. Hence the result of the previous paragraph yields $J^{\prime \prime}=1$ and so $J^{\prime}$ is faithful on $z_{2}^{L_{1}}$. So, if we set $\Delta=z_{2}^{L_{1}}$, then $\left(J^{\prime} L_{1}\right)^{\Delta}$ $=J^{\prime \Delta} \times K_{1}^{\Delta} \times K_{1}^{t \Delta}$. Since $|\Delta|=\left|J^{\prime} L_{1}: C_{J^{\prime} L_{1}}\left(z_{2}\right)\right|,\left|C_{J^{\prime} L_{1}}\left(z_{2}\right)\right|=\left|J^{\prime}\right|\left|C_{L_{1}}\left(z_{2}\right)\right|$. Then by the result obtained in the first paragraph we have $C_{J^{\prime} L_{1}}\left(z_{2}\right)=J^{\prime} C_{L_{1}}\left(z_{2}\right)$. Since $J^{\prime \Delta}$ is normal and contained in a one-point-stabilizer of the transitive permutation group $\left(J^{\prime} L_{1}\right)^{4}$, we have $J^{\prime \Delta}=1^{\Delta}$ and $C_{J_{2} L_{1}}\left(z_{2}\right)=C_{L_{1}}\left(z_{2}\right)$. Therefore $\left|z_{2}^{J_{2} L_{1}}\right|=\left(q^{2}-1\right) q^{2}\left(q^{2}+1\right)^{2}$ or $\left(q^{2}-1\right) q^{2}\left(q^{2}+1\right)$ according as $C_{M_{1}}\left(z_{2}\right) \simeq S L_{2}(q) \times S L_{2}(q)$ or $S L_{2}\left(q^{2}\right)$. But $\left(q^{2}-1\right) q^{2}\left(q^{2}+1\right)^{2}>q^{8}$. Consequently $C_{J_{2} L_{1}}\left(z_{2}\right) / F_{1} \simeq S L_{2}\left(q^{2}\right)$ and the proof is complete. 
(4.3) $z_{1}$ and $z_{2}$ are the representatives of $N\left(F_{1}\right)$-conjugacy classes of the elements of $F_{1}^{\#}$ and the fusion is controled by $J_{2} L_{1}$. Furthermore $\left|z_{1}^{N\left(F_{1}\right)}\right|=$ $\left(q^{2}-1\right)\left(q^{2}+1\right)^{2}$ and $\left|z_{2}^{N\left(F_{1}\right)}\right|=\left(q^{2}-1\right)^{2} q^{2}\left(q^{2}+1\right)$. have

Proof. By (3.17.4) and (4.1.2) $\left|\bigcup_{x \in L_{1}}\left(E^{x}\right)^{\sharp}\right|=\left(q^{2}-1\right)\left(q^{2}+1\right)$. By (4.2) we

$$
\left|z_{1}^{L_{1}}\right|=\left(q^{2}-1\right)\left(q^{2}+1\right)^{2} \text { and }\left|z_{2}^{J_{2} L_{1}}\right|=\left(q^{2}-1\right)^{2} q^{2}\left(q^{2}+1\right) .
$$

Hence $L_{1}$ has one orbit of length $\left(q^{2}-1\right)\left(q^{2}+1\right)^{2}$ and $q^{2}-1$ orbits of length $\left(q^{2}-1\right) q^{2}\left(q^{2}+1\right)$ on $F_{1}^{*}$, and the orbits of length $\left(q^{2}-1\right) q^{2}\left(q^{2}+1\right)$ are permuted transitively by $J_{2}$. Since $L_{1} \triangleleft N\left(F_{1}\right)$, the assertion follows.

(4.4) Set $U \cap M_{1}=U_{0}$. Then the following statements hold.

(1) $C_{F_{1}}(a)=Z(U)$ for $a \in U_{0}^{\#}$ and $\left|C_{F_{1}}(b)\right|=q^{4}$ for $b \in U_{0} U_{0}^{t}-U_{0}-U_{0}^{\iota}$. In particular $C_{F_{1}}(a, b)=E$.

(2) $\mathscr{E}\left(C_{R}(a)\right)=\left\{Z(U) U_{0}, E U_{0} U_{0}^{t}\right\}$ and $\mathscr{E}\left(C_{R}(b)\right)=\left\{V, E U_{0} U_{0}^{t}\right\}$, where $V=$ $C_{R}\left(C_{F_{1}}(b)\right) \cap C_{R}(b)$.

(3) $C_{R}(x, b)=V$ for any $x \in C_{F_{1}}(b)-E$. Also $\mathscr{E}\left(C_{R}(c)\right)=\left\{V, E U_{0} U_{0}^{\iota}\right\}$ for any $c \in V^{\sharp} \cap U_{0} U_{0}^{t}$. $=q^{10}$.

(4) If $x \in Z(U)-E$, then $C_{R}(x)=U$. If $x \in F-Z(U)-Z\left(U^{t}\right)$, then $\left|C_{R}(x)\right|$

Proof. Suppose $C_{F_{1}}(a)>Z(U)$. Then since $C_{F_{1}}(a)=C_{F_{1}}\left(a^{x}\right)$ for any $x \in K_{1}^{t}$, we have $C_{F_{1}}(a)=F_{1}$ by $(3.17 .1,3)$ and $(4.1 .2)$. This yields that $C_{R}(a)$ $=R$, since $R=F_{1}\left(U_{0} \times U_{0}^{t}\right)$, which is against (3.9.1). Hence $C_{F_{1}}(a)=Z(U)$. By the structure of $M_{1}$ the elements of $U_{0} U_{0}^{t}-U_{0}-U_{0}^{t}$ are conjugate mutually in $N_{M_{1}}(R)$. So we may assume that $b \in C_{N_{1}}\left(z_{2}\right)<C_{M_{1}}(z)$. Now the proof of (4.2) shows that $C_{J_{1} J_{2}}\left(L_{1} / F_{1}\right)$ acts on $F_{1}^{\#}$ fixed-point-freely. Therefore if we denote $X=C_{J_{1} J_{2}}\left(L_{1} / F_{1}\right)$, then $\left\langle z_{2}^{X}\right\rangle E \leq C_{F_{1}}(b)$ and $\left|\left\langle z_{2}^{X}\right\rangle E\right| \geq q^{4}$. By (4.1.1) $C_{F_{1}}(b) \leq F$. Hence, if $\left|C_{F_{1}}(b)\right|>q^{4}$, then by (3.17.5) $Z(U) \cap C_{F_{1}}(b)>E$. Let $c \in Z(U) \cap C_{F_{1}}(b)$ $-E$ and let $b=b_{1} b_{2}$ with $b_{1} \in K_{1}$ and $b_{2} \in K_{2}^{t}$. Since $b c=c b$ and $b_{1} c=c b_{1}$, we have $b_{2} c=c b_{2}$, which contradicts the first assertion. Thus (1) holds. Also it is obtained that $C_{F_{1}}(b) \cap Z(U)=E$ and (4) follows immediately.

By (1) $C_{R}(a)=\left(Z(U) U_{0}\right)\left(E U_{0} U_{0}^{t}\right), Z(U) U_{0} \cap E U_{0} U_{0}^{t}=E U_{0}$ and $C_{R}(a, d)=$ $E U_{0} U_{0}^{t}$ for any $d \in E U_{0} U_{0}^{t}-Z(U) U_{0}$. This implies that $\mathscr{E}\left(C_{R}(a)\right)=$ $\left\{Z(U) U_{0}, E U_{0} U_{0}^{t}\right\}$. Now we may take $b=z_{2}^{r_{2}}$. Then $C_{F_{1}}(b)=\left\langle z_{2}^{X}\right\rangle E$. Since $C_{F_{1}}(b) \cap Z(U)=E, C_{L_{1}}(x) / F_{1} \simeq S L_{2}\left(q^{2}\right)$ for any $x \in C_{F_{1}}(b)-E$ by (3.17.5) and (4.2). Hence we have $C_{R}(x) \in \operatorname{Syl}_{2}\left(C_{L_{1}}(x)\right)$ by the structure of $M_{1}$. Then $C_{F_{1}}(y)=$ $\left\langle x^{X}\right\rangle E=\left\langle z_{2}^{X}\right\rangle E$ for any $y \in C(x) \cap U_{0} U_{0}^{t}$, since $C(x) \cap\left(M_{1} \cap K_{1}\right) \cap U_{0} U_{0}^{t}=C(x) \cap$ $\left(M_{1} \cap K_{1}^{t}\right) \cap U_{0} U_{0}^{t}=1$ by the proof of (4.2). Hence $V=C_{R}\left(C_{F_{1}}(b)\right) \cap C_{R}(b)$ is abelian. So in order to prove (2) and (3) we only show that $\mathscr{E}\left(C_{R}(b)\right)=$ $\left\{V, E U_{0} U_{0}^{t}\right\}$. Let $x \in I\left(C_{R}(b)\right)$ and let $x=x_{1} x_{2} x_{3}$, where $x_{1} \in C_{F_{1}}(b), x_{2} \in U_{0}$ and 
$x_{3} \in U_{0}^{t}$. Furthermore we can choose $x_{3}^{\prime}$ in $U_{0}^{t}$ such that $x_{2} x_{3}^{\prime} \in C\left(x_{1}\right)$. Then $1=\left(x_{1} x_{2} x_{3}\right)^{2}=\left(x_{1} x_{2} x_{3}^{\prime} x_{3}^{\prime} x_{3}\right)^{2}=\left(x_{1} x_{3}^{\prime} x_{3}\right)^{2}$, which yields that $x_{3}^{\prime} x_{3}=1$ or $x_{1} \in E$. This gives the claim.

(4.5) (1) $I\left(F_{2}\right)=\bigcup_{x \in L_{2}}\left(F^{\frac{m}{*}}\right)^{x}$.

(2) If $x \in I(R)$, then $x$ is either conjugate to $z_{1}$ or to $z_{2}$ in $L_{1} \cup L_{2}$.

(3) $t^{G} \cap R T=t^{R}=I(t R)$.

(4) $\left|C_{F_{1}}(u)\right|=q^{4}$ and $\left|C_{F_{1}}(u t)\right|=q^{6}$, where the involution $u$ defied in Section 2 is chosen to normalize each component of $M_{1}$.

Proof. By (3.9.3) and (3.11.2) $F \cap F^{r_{2}}=E$. Hence $F \cap F^{x}=E$ for any $x \in L_{2}-N_{L_{2}}(R)$ by the structure of $L_{2} / F_{2}$. Therefore $\left|\bigcup_{x \in L_{2}}\left(F^{\#}\right)^{x}\right|=q^{\beta}+q^{3}-q^{4}-1$. By $(4.1) C_{R}(y) \leq F_{2}$ for any $y \in\left(U_{0} U_{0}^{t}\right)^{\sharp}$. Hence applying (4.4.1), we have $\left|I\left(F_{2}\right)\right|=\left(q^{4}-1\right) q^{4}+q^{6}-1=q^{8}+q^{6}-q^{4}-1$. Thus (1) holds. Then (2) follows from (4.3).

By (1) for any $x \in I(R) m(C(x)) \geq 8 n \geq 4 n+1=m(C)$, so $t$ does not fuse into $R$. Since $R T / R \simeq T / P, m(R T / R)=1$ or 2 . If $m(R T / R)=1$, then $I(R T-R)$ $=I(t R)$ and we can apply (19.4) of [4] to $R / F_{1}$ and $F_{1}$ to obtain $t^{R}=I(t R)$. If $m(R T / R)=2$, then $I(R T-R)=I(t R) \cup I(t u R) \cup I(u R)$. Set $X=N_{L_{1}}(R) T$ and set $\Omega=E^{\sharp}$. Then $\left(x^{u}\right)^{\Omega}=\left(x^{q}\right)^{\Omega}$ for any $x \in J_{1}$. Since $X$ satisfies the assumption of (1.3), we have $\left(x^{t}\right)^{\Omega}=\left(x^{q}\right)^{\Omega}$. So $\left(x^{t \imath}\right)^{\Omega}=x^{\Omega}$ for any $x \in J_{1}$, which implies $t u \in C(E)$. By (3.17) $F_{1}=E \times Y \times Y^{t} \times E^{r_{1}}$, where $Y=Z(U) \cap Z\left(U^{t r_{1}}\right)$. Since $r_{1} u=u r_{1}, E, E^{r_{1}} \leq C(t u)$. Since $t u$ interchanges $Y$ and $Y^{t}, C_{Y Y^{t}}(t u)=$ $\left\{z z^{t u} \mid z \in Y\right\}$. Consequently $\left|C_{F_{1}}(t u)\right|=q^{6}$. Then applying (19.4) of [4] to $R / F_{1}$, we obtain that any element of $I(t u R)$ is $R$-conjugate to one of $I\left(t u F_{1}\right)$. Thus for any $x \in I(t u R),\left|C_{F_{1}}(x)\right|=q^{6}$. Since $m(C)=4 n+1$ by (3.1.1), $t$ does not fuse into $I(i u R)$. Next we inspect $C_{F_{1}}(u)$. By (3.17.2) $E \times Y \times Y^{t}=F$. So $Z_{2}(P)$ $=Z(P) C_{Y^{t}}(t)$ and $\left|C_{Y Y^{t}}(t, u)\right|=q$. Hence $\left|C_{Y}(u)\right|=\left|C_{Y^{t}}(u)\right|=q$. Since $C_{E}(t)=$ $C_{E}(u)$, we get $\left|C_{F_{1}}(u)\right|=q^{4}$ and $I(u R)=u^{R}$ as above. This gives (4).

Then in order to prove (3) it is enough to show that $t$ is not conjugate to $u$. Suppose false. Then, since $B_{1}=J r(T), C_{F_{1}}(u)\langle u\rangle$ is conjugate to $B_{1}$. But $N_{c}\left(A_{1}\right)^{(\infty)}=A_{1} N_{1}$, while $C_{L_{1}}(u) / F_{1} \simeq S L_{2}(q) \times S L_{2}(q)$, a contradiction. Thus (3) is proved.

(4.6) Let $b \in U_{0} U_{0}^{t}-U_{0}-U_{0}^{t}$ and let $b_{i}=b^{x^{i}}$, where $\langle x\rangle=J_{1} \cap K_{1}$. Set $\mathscr{E}\left(C_{R}\left(b_{i}\right)\right)=\left\{V_{i}, E U_{0} U_{0}^{t}\right\}$ and set $B=Z(U) U_{0}$. Then $V_{i}, i=1,2, \cdots, q^{2}-1, B$ and $B^{t}$ are normal abelian subgroup of $L_{2}$ contained in $F_{2}$. Moreover $L_{2} / E$ acts on $B / E$ in the same way as the natural action of $S L_{2}\left(q^{2}\right)$ on a 2-dimensional vector space over $G F\left(q^{2}\right)$.

Proof. First we will show that $\left\{V_{i}-E, B-E, B^{t}-E \mid i=1, \cdots, q-1\right\}$ makes a partition of the involutions of $F_{2}-E$. By (4.4.1) $F_{1} \cap V_{i} \cap V_{j} \leq$ 
$C_{R}\left(b_{i}, b_{j}\right)=C_{R}\left(b^{i}, b_{i} b_{j}\right)=E$ if $i \neq j$, since $b_{i} b_{j} \in F_{1} U_{0}$. Then by (4.4.3) $V_{i} \cap V_{j}=$ $E$. Similarly we have $B \cap V_{i}=B^{t} \cap V_{i}=E$ for any $i$ by (4.4.4). Then counting argument gives the claim as in (4.5.1). In particular we may assume that $\left\langle z_{2}, z_{2}^{r_{2}}\right\rangle\left\langle V_{1}\right.$. By (4.4.3) $C_{R}\left(z_{2}, z_{2}^{r_{2}}\right)=V_{1}$ and $C_{R}\left(z_{2}\right)=F_{1} V_{1}$. Hence by (4.1.1) $V_{1} \triangleleft R$. Therefore we have $V_{1} \triangleleft\left\langle R, R^{r_{2}}\right\rangle=L_{2}$. Since $J_{1}$ normalizes $L_{2}$ by the proof of (3.14), this implies that all the $V_{i}^{\prime}$ 's are normal in $L_{2}$. Hence by (4.4.4) $U_{0} \cup U_{0}^{t} \subseteq B^{x} \cup B^{t x}$ for any $x \in L_{2}$ and by (4.4.2) $B^{x}=B$ or $B^{t}$. By (3.13.3) $L_{2} / E$ has no subgroup of index 2 , which yields that $B^{x}=B$. Since $|(B / E) \cap(F / E)|=q^{2}$, the last assertion follows from $(1 K)$ of [12].

DeFinition. $G_{0}=\left\langle L^{G}\right\rangle$.

(4.8) (1) $F_{1}=\operatorname{Jr}(R)$.

(2) $R \in \operatorname{Syl}_{2}\left(G_{0}\right)$.

(3) $G_{0}$ has just two conjugacy classes of involutions, which are represented by $z_{1}$ and $z_{2}$. Furthermore $C_{R}\left(z_{2}\right)$ is a Sylow 2-subgroup of $C_{G_{0}}\left(z_{2}\right)$.

(4) $B$ and $B^{t}$ are weakly closed in $R$ with respect to $G_{0}$.

Proof. (1) follows from (4.1.1) and (4.7). By (4.5.3) $N(R T)=$ $R T(C \cap N(R T))$. Hence $R T \in \operatorname{Syl}_{2}(G)$. Since $R T / R \simeq T / P$ is abelian, we have $t \notin O^{2}(G)$ by (1.5). By (3.13.3) $R<O^{2}(G)$. Suppose that $R \notin \operatorname{Syl}_{2}\left(O^{2}(G)\right)$. Then $\langle u\rangle R$ or $\langle t u\rangle R$ is contained in $O^{2}(G)$. Hence there exists an element $x$ in $R$ such that $x=u^{g}$ or $(t u)^{g}$ for some $g \in G$. By (4.5.1) and (4.5.4) we have

$$
\begin{aligned}
& m(C(y)) \geq 8 n \quad \text { if } y \in I(R), \\
& m\left(C_{R T}(y)\right) \leq 6 n+1 \quad \text { if } y \in I(R T-R) .
\end{aligned}
$$

So in any case we may take $C_{(R T) g}(x) \leq R T$. Then $t^{g} \in R T$. Hence by (4.5.3) we may assume $g \in C$. This implies that $u$ or $t u$ fuses into $P$ in $C$, which does not occur. Thus $R \in \operatorname{Syl}_{2}\left(O^{2}(G)\right)$. By (4.5.2) $\left\langle L_{1}, L_{2}\right\rangle \leq G_{0}$. Therefore $R \in \operatorname{Syl}_{2}\left(G_{0}\right)$ and also the first statement of (3) holds. Then by (4.2) and (4.4.1) we have $C_{R}\left(z_{2}\right) \in \operatorname{Syl}_{2}\left(C_{G_{0}}\left(z_{2}\right)\right)$. By (4.6) $B^{\sharp} \subseteq z_{1}^{G_{0}}$ and $V_{i}^{\#}, F^{\#} \subseteq z_{1}^{G_{0}}$. So by (4.7), if $B^{x}<R$, then $B^{x}=B$ or $B^{t}$, or else $B^{x}<F_{1}$. Suppose that $B^{x}<F_{1}$. Then $B^{x} \cap F \subseteq Z(U) \cup Z\left(U^{t}\right)$ by (3.17.5). Since $\left|B^{x} \cap F\right| \geq q^{4}$, we may assume that $B^{x} \cap F=Z(U)$ without loss of generality. Now similarly $B^{x} \cap F^{r_{1}} \subseteq Z\left(U^{r_{1}}\right)$ $\cup Z\left(U^{r_{1} t}\right)$. If $B^{x} \cap F^{r_{1}}=Z\left(U^{r_{1}}\right)$, then $B^{x}=Z(U) Z\left(U^{r_{1}}\right)=F_{1}$ by (3.17.1), a contradiction. If $B^{x} \cap F^{r_{1}}=Z\left(U^{r_{1} t}\right)$, then $B^{x}=Z(U) Z\left(U^{r_{1} t}\right)$. By (4.1.4) this yields that $B^{x}=Z_{2}\left(U U^{r_{1} t}\right)$, also a contradiction. Thus $B^{x}=B$ or $B^{t}$. Then by a theorem of Burnside we have $x \in N_{G_{0}}(R)$ and by (4.6) $B^{x}=B$. Thus $B$ is weakly closed in $R$ with respect to $G_{0}$. By symmetry $B^{t}$ is weakly closed.

(4.9) $L_{2} \triangleleft N\left(F_{2}\right)$.

Proof. Set $\overline{N\left(F_{2}\right)}=N\left(F_{2}\right) / F_{2}$ and set $X=N\left(F_{2}\right) \cap G_{0}\langle t\rangle$. By (4.8) 
$Q \in \operatorname{Syl}_{2}\left(C_{X}(t)\right)$. Hence by (3.8) and (3.10) $\bar{N}_{2}$ is a standard subgroup of $\bar{X}$ and $\langle\bar{t}\rangle$ is a Sylow 2-subgroup of $C_{\bar{X}}\left(\bar{N}_{2}\right)$. So, as in (3.12), we have $\left\langle\bar{N}_{2}^{\bar{X}}\right\rangle \simeq S L_{2}(q) \times$ $S L_{2}(q)$ or $S L_{2}\left(q^{2}\right)$. Since $\bar{T}$ normalizes $\bar{N}_{2},\left\langle\bar{N}_{2}^{\overline{N\left(F_{2}\right)}}\right\rangle=\left\langle\bar{N}_{2}^{\bar{X}}\right\rangle$. Therefore. $\bar{L}_{2}=\left\langle\overline{\left.N_{2}^{N\left(F_{2}\right)}\right\rangle} \triangleleft \overline{N\left(F_{2}\right)}\right.$.

Now we can appeal to an argument in [12] for the conclusion of this section. $(4.10,11,12$ and 13$)$ below refer to $(4 N, O, P, S)$ of [12].

(4.10) $\quad N_{G_{0}}(E)=N_{G_{0}}\left(F_{2}\right) O\left(N_{G_{0}}(E)\right)$.

Proof. Set $\overline{N_{G_{0}}(E)}=N_{G_{0}}(E) / O\left(N_{G_{0}}(E) \bmod E\right)$ and let $X$ be the normal closure of $F_{2}$ in $N_{G_{0}}(E)$. Then by (4.1.1) and (4.8.2) we can appeal to $(1 H)$ of [12] to obtain that $F_{2}$ is strongly closed in $R$ with respect to $N_{G_{0}}(E)$. Hence by the result of [10] we have $F_{2} \in \operatorname{Syl}_{2}(X)$, and either $\bar{X}=\bar{F}_{2}$ or $\bar{X}=O_{2}(\bar{X}) \bar{X}_{1}$ $\cdots \bar{X}_{k}$, where $\bar{X}_{i}$ is a simple Goldschmidt group. Suppose the latter holds. Since $\bar{t}$ normalizes $\bar{X}$ and since $\mathscr{E}(\langle t\rangle E)=\{E, Z(Q)\}$,

$$
C(\bar{t}) \cap \bar{F}_{2} \cap \bar{X}_{1} \bar{X}_{1}^{t}=\bar{A}_{2} \cap \bar{X}_{1} \bar{X}_{1}^{t} \neq 1 \text {. }
$$

Let $\bar{x}_{i} \in\left(\bar{A}_{2} \cap \bar{X}_{i} \bar{X}_{i}^{t}\right)^{\#}$. If there exist $\bar{X}_{1}, \bar{X}_{2}$ and $\bar{X}_{3}$ such that $\bar{X}_{1} \bar{X}_{1}^{t} \neq \bar{X}_{2} \bar{X}_{2}^{t} \neq$

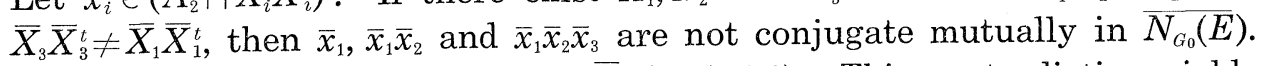
But $\bar{N}_{L}(E)$ has only two orbits on $\bar{A}_{2}^{\#}$ by (2.2.2). This contradiction yields that $E(\bar{X})$ has at most four components. Therefore $\overline{C_{L}(E)^{\prime}}$ normalizes each $\bar{X}_{i}$, since $\bar{C}_{L}(E)^{\prime}$ is perfect. In particular it normalizes $\bar{F}_{2} \cap \bar{X}_{i}$ which is a Sylow 2-subgroup of $\bar{X}_{i}$. Consequently $\overline{C_{L}(E)^{\prime}}$ centralizes each $\bar{X}_{i}$. But this yields that $\overline{C_{L}(E)^{\prime}}$ centralizes $\bar{A}_{2}$, a contradiction. Therefore $\bar{F}_{2} \triangleleft N_{G_{0}}(E)$. Then, since $E=Z\left(F_{2}\right)$, we have $F_{2} O\left(N_{G_{0}}(E)\right) \triangleleft N_{G_{0}}(E)$ and the Frattini argument completes the proof.

Definition. Let $C_{i}=C_{G_{0}}\left(z_{i}\right), i=1,2$.

(4.11) $C_{1}=N_{C_{1}}(E) O\left(C_{1}\right)$ and $C_{1}$ is 2-constrained.

Proof. Suppose an element $z$ of $E$ is $C_{1}$-conjugate to an element $x$ of $R-E$. Then by (3.13.2), (4.1.1) and (4.5.1) we may take $x$ in $F_{1}-E$. Since $F_{1}$ is weakly closed in $R, z$ and $x$ are conjugate in $N_{C_{1}}\left(F_{1}\right)$. But by (3.17.4) we have $N_{C_{1}}\left(F_{1}\right) \leq N(R) \leq N(E)$, a contradiction. So $E$ is strongly closed in $R$ with respect to $C_{1}$.

Let $X=\left\langle E^{C_{1}}\right\rangle$ and let $\bar{C}_{1}=C_{1} / O\left(C_{1}\right)$. Then by the result of [10] we have either $\bar{X}=\bar{E}$ or $\bar{X}=O_{2}(\bar{X}) \bar{X}_{1} \cdots \bar{X}_{k}$, where $\bar{X}_{i}$ is a quasisimple Goldschmidt group. Furthermore we have $E=\Omega_{1}(R \cap X)$. If $E<R \cap X$, then by (3.9.1) $E<F \cap X \leq \Omega_{1}(R \cap X)$, a contradiction. Hence $E \in \operatorname{Syl}_{2}(X)$. If $\bar{X} \neq \bar{E}$, then $\bar{L}_{2}$ normalizes each $\bar{X}_{i}$ by (3.13.2). Now by (3.17.3) $N_{L_{1}}(R)$ acts on $E^{\sharp}$ transitively. Hence $E \leq R^{\prime}$ and so $L_{2}$ is perfect by (3.13.3). Therefore $\bar{L}_{2}$ centralizes 
each $\bar{X}_{i}$. Then $\bar{E} \cap \bar{X}_{i} \leq \bar{L}_{2} \cap \bar{X}_{i} \leq Z\left(\bar{X}_{i}\right)$, which does not hold. Hence $\bar{X}=\bar{E}$, and it follows that $C_{1}=N_{C_{1}}(E) O\left(C_{1}\right)$. Since $C_{R}\left(F_{2}\right)<F_{2}<R$ by (4.1.1), $N_{G_{0}}\left(F_{2}\right)$ is 2-constrained and so is $N_{G_{0}}(E)$ by (4.10). Thus $C_{1}$ is 2-constrained.

$$
C_{2}=N_{C_{2}}\left(F_{1}\right) O\left(C_{2}\right) \text { and } C_{2} \text { is 2-constrained. }
$$

Proof. By (4.8.3) $C_{R}\left(z_{2}\right)=F_{1} V_{1} \in \operatorname{Syl}_{2}\left(C_{2}\right)$ and $\mathscr{E}\left(C_{R}\left(z_{2}\right)\right)=\left\{V_{1}, F_{1}\right\}$, where $V_{1}$ is defined in the proof of (4.6). Hence we can apply $(1 H)$ of [12] to obtain that $F_{1}$ is strongly closed in $C_{R}\left(z_{2}\right)$ with respect to $C_{2}$. Let $X$ be the normal closure of $F_{1}$ in $C_{2}$ and set $\bar{C}_{2}=C_{2} / O_{2}\left(C_{2}\right)$. Then as in (4.10) we have $F_{1} \in$ $\operatorname{Syl}_{2}(X)$ and either $\bar{X}=\bar{F}_{1}$ or $\bar{X}=O_{2}(\bar{X}) \bar{X}_{1} \cdots \bar{X}_{k}$, where $\bar{X}_{i}$ is a simple Goldschmidt group. Suppose that $\bar{X} \neq \bar{F}_{1}$. Then by (4.11) $C_{1} \cap C_{2}$ is 2 -constrained and so is $\overline{C_{1} \cap C_{2}}$. Hence $C_{\bar{X}}(\bar{z})$ is 2-constrained for any $z \in E^{\#}$. So, if we set $\bar{z}=$ $\bar{x} \bar{y}_{1} \cdots \bar{y}_{k}$, where $\bar{x} \in O_{2}(\bar{X})$ and $\bar{y}_{i} \in \bar{X}_{i}$, then it follows that $\bar{y}_{i} \neq \overline{1}$ for all $i$. Thus $|\bar{E}| \leq\left|\bar{F}_{1} \cap \bar{X}_{i}\right|$. Since all the elements of $\left(\bar{F}_{1} \cap \bar{X}_{i}\right)^{\#}$ are conjugate mutaually in $N_{\bar{X}_{i}}\left(\bar{F}_{1} \cap \bar{X}_{i}\right), F_{1}$ contains at least $\left(q^{2}-1\right)^{k}$ elements conjugate to z. Then by (4.3) $\left(q^{2}-1\right)^{k} \leq\left(q^{2}-1\right)\left(q^{2}+1\right)^{2}$. Hence $k \leq 3$ and as in (4.10) we obtain that $\overline{C_{L}\left(z_{2}\right)^{\prime}}$ centralizes each $\bar{X}_{i}$ by (2.2.3). Then $\bar{z}_{1} \in C_{\bar{X}}(E(\bar{X}))$. Since $\overline{C_{1} \cap C_{2}}$ is 2-constrained, this can not occur. Thus we have $\bar{X}=\bar{F}_{1}$ and the assertion follows easily.

(4.13) $\quad G_{0} \simeq L_{4}\left(q^{2}\right)$.

Proof. By (4.8.3), (4.11) and (4.12) the centralizer of any involution in $G_{0}$ is 2-constrained. Furthermore $S C N_{3}(2)$ is not empty, as $F_{1} \in S C N_{3}(2)$. Thus by a result of [14] we have $O\left(C_{1}\right)=O\left(C_{2}\right)=1$, since we assumed $O(G)=1$ at the beginning of Section 3. Hence $L_{2} \triangleleft C_{1}$ by (4.9). Suppose that $B^{g}<C_{1}$ for some $g \in G_{0}$. Then $B^{g}<L_{2}$. Hence $B^{g h}<R$ for some $h \in L_{2}$, and so by (4.8.4) $B^{g h}=B$. Consequently we have $B^{g}=B$ by (4.6). Since $B^{\sharp} \subseteq z_{1}^{G_{0}}$ by (4.6), for any $a \in B^{\sharp} B$ is weakly closed in $C_{G_{0}}(a)$ with respect to $G_{0}$. Since $G_{0}$ is simple (cf. Lemma 2.7 of [2]), it follows that $G_{0}=\left\langle B^{G_{0}}\right\rangle$. Thus we can appeal to the result of Timmesfeld [19] to conclude that $G_{0}$ is isomorphic to $L_{4}\left(q^{2}\right)$

\section{§5. Case for $U_{4}(q) \times U_{4}(q)$}

In this section we assume the following and we will obtain that $\left\langle L^{G}\right\rangle$ $\simeq U_{4}(q) \times U_{4}(q)$ under the hypothesis made at the beginning of Section 3 .

HypothesIS. $L_{2} / F_{2} \simeq S L_{2}(q) \times S L_{2}(q)$.

Definition. Set $L_{2}=K_{2} K_{2}^{t}$, where $K_{2} \triangleleft L_{2}$ and $K_{2} / F_{2} \simeq S L_{2}(q)$. 
(5.1) (1) $\quad F_{1}=\left(Z(U) \cap Z\left(U^{r_{1}}\right)\right) \times\left(Z\left(U^{t}\right) \cap Z\left(U^{r_{1} t}\right)\right)$.

(2) $K_{1}$ acts on $Z(U) \cap Z\left(U^{r_{1}}\right)$ trivially and on $Z\left(U^{t}\right) \cap Z\left(U^{r_{1} t}\right)$ irreducibly.

(3) $L_{1}=K_{1}^{\prime} \times K_{1}^{\prime t}$.

Proof. Suppose $|Z(U)|=q^{4}$. Then by (3.16.2) we can apply (3.17.3) to obtain that $J_{1} \cap K_{1}$ acts on $\left(F_{1} / F\right)^{\ddagger}$ transitively. Since $R / F_{2} \simeq F_{1} / F, C_{F_{1} / F}\left(J_{2}\right.$ $\left.\cap K_{2}\right) \neq 1$. Then by (3.14) $C_{F_{1} / F}\left(J_{2} \cap K_{2}\right)$ is $J_{1} \cap K_{1}$-invariant. Hence this yields that $C_{F_{1} / F}\left(J_{2} \cap K_{2}\right)=F_{1} / F$, so $C_{R / F_{2}}\left(J_{2} \cap K_{2}\right)=R / F_{2}$. This can not occur by the structure of $L_{2} / F_{2}$. Thus we have $|Z(U)|>q^{4}$ and $Z(U) \cap Z\left(U^{r_{1}}\right) \neq 1$. Since $\left\langle U, U^{r_{1}}\right\rangle=K_{1}, K_{1} \leq C\left(Z(U) \cap Z\left(U^{r_{1}}\right)\right)$. If $\left|Z(U) \cap Z\left(U^{r_{1}}\right)\right|<q^{4}$, then by Lemma $4 \mathrm{~B}$ of [8] $K_{1}^{t} \leq C\left(Z(U) \cap Z\left(U^{r_{1}}\right)\right)$. But this contradicts (3.5.1). On the other hand we have $Z(U) \cap Z\left(U^{r_{1}}\right) \cap Z\left(U^{t}\right) \cap Z\left(U^{t r_{1}}\right)=E \cap E^{r_{1}}=1$ by (3.11.1). Thus (1) holds. Also (2) follows from (1). Then we have

$$
K_{1}=\left(Z(U) \cap Z\left(U^{r_{1}}\right)\right) \times\left(\left(Z\left(U^{t}\right) \cap Z\left(U^{r_{1} t}\right)\right) \cdot\left(K_{1} \cap M_{1}\right)\right) .
$$

Hence Gaschütz's theorem yields that $K_{1}^{\prime}=\left(Z\left(U^{t}\right) \cap Z\left(U^{r_{1} t}\right)\right) \cdot\left(K_{1} \cap M_{1}\right)$ and so $L_{1}=K_{1}^{\prime} \times K_{1}^{\prime t}$.

(5.2) Set $W=R \cap K_{1}^{\prime}, W_{i}=\left[W, I_{i}\right], i=1,2$. Then the following statements hold.

(1) $E=Z(W) \times Z\left(W^{t}\right), F=Z_{2}(W) \times Z_{2}\left(W^{t}\right)$ and $R=W \times W^{t}$.

(2) $F_{1}=W_{1} \times W_{1}^{t}$ and $F_{2}=W_{2} \times W_{2}^{t}$.

(3) $W_{1}=O_{2}\left(K_{1}^{\prime}\right)=Z\left(U^{t}\right) \cap Z\left(U^{r_{1} t}\right)$.

Proof. (1) is clear by (5.1.3). By (3.9.3) $F_{1}=\left[R, I_{1}\right]=\left[W \times W^{t}, I_{1}\right]=$ $\left[W, I_{1}\right] \times\left[W^{t}, I_{1}\right]=W_{1} \times W_{1}^{t}$. Also $F_{2}=\left[R, I_{1}\right]=W_{2} \times W_{2}^{t}$. Thus (2) holds. Then, since $\left|W_{1}\right|=\left|Z\left(U^{t}\right) \cap Z\left(U^{r_{1} t}\right)\right|$ and $W_{1} \leq F_{1} \cap K_{1}^{\prime}$, we have (3).

$$
L_{2}=K_{2}^{\prime \prime} \times K_{2}^{\prime \prime t} \text { and } W \text { is a Sylow 2-subgroup of } K_{2}^{\prime \prime} \text { or } K_{2}^{\prime \prime t} \text {. }
$$

Proof. By (3.14) $J_{2} / C_{J_{2}}\left(K_{1}^{\prime} / W\right)$ acts on $K_{1}^{\prime} / W$ as an inner automorphism. Since $J_{2} \simeq Z_{q-1} \times Z_{q-1}, \quad\left|C_{J_{2}}\left(K_{1}^{\prime} / W_{1}\right)\right| \geq q-1$. Set $J=C_{J_{2}}\left(K_{1}^{\prime} / W_{1}\right)$. Suppose $J \not Z K_{2} \cup K_{2}^{t}$. Then $C_{R / F_{2}}(J)=1$ and so $C_{F_{1} / F}(J)=1$. Hence $C_{F_{1}}(J) \leq F$ and by (3.13.2) and (5.2) we have $1 \neq C_{W_{1}}(J)<W_{1}$. But this contradicts (5.1.2). Therefore $J \subseteq K_{2} \cup K_{2}^{t}$. So we may assume that $J=J_{2} \cap K_{2}^{t}$. Then $C_{W_{1}}(J)$ $\neq 1$, which yields that $\left[W_{1}, J\right]=1$. Since $J$ stabilizes a normal series $W \triangleright W_{1} \triangleright 1$, we have $[W, J]=1$. Set $V=K_{2} \cap R$. Then

$$
V^{t}=F_{2}[R, J]=\left(W_{2} \times W_{2}^{t}\right)\left([W, J] \times\left[W^{t}, J\right]\right) \leq W_{2} \times W_{2}^{t}\left[W^{t}, J\right] .
$$

Comparing the orders of both sides, we have $V^{t}=W_{2} \times W^{t}$. By symmetry $V=W \times W_{2}^{t}$. As a consequence $Z(V)=E$ and $Z_{2}(V)=Z_{2}(W) \times W_{2}^{t}$. Set $\bar{L}_{2}$ $=L_{2} / E$. By (3.9.2) $\left[\overline{I \cap J}_{2}, \bar{F}_{2}\right]=\bar{F}_{2}$, so in particular $\left[\bar{J}_{2}, \bar{W}_{2}\right]=\bar{W}_{2}$. Since $J_{2}=$ 
$\left(J_{2} \cap K_{2}\right) \times J$ and $[\bar{J}, \bar{W}]=\overline{1}$, we have $\left[\overline{J_{2} \cap K_{2}}, \bar{W}_{2}\right]=\bar{W}_{2}$. Notice that $|Z(\bar{V})|=q^{6}$ and that $K_{2}=\left\langle V, V^{r_{2}}\right\rangle$. Hence $\left|Z(\bar{V}) \cap Z\left(\bar{V}^{r_{2}}\right)\right| \geq q^{4}$ and $K_{2} \leq C\left(Z(\bar{V}) \cap Z\left(\bar{V}^{r_{2}}\right)\right)$. Therefore we have

$$
\begin{aligned}
Z(\bar{V}) \cap Z\left(\bar{V}^{r_{2}}\right) \cap \bar{W} & =Z(\bar{V}) \cap Z\left(\bar{V}^{r_{2}}\right) \cap \bar{W} \cap \bar{F}_{2} \\
& =Z(\bar{V}) \cap Z\left(\bar{V}^{r_{2}}\right) \cap \bar{W}_{2}=\overline{1} .
\end{aligned}
$$

Hence $\bar{V}=\left(Z(\bar{V}) \cap Z\left(\bar{V}^{r_{2}}\right)\right) \times \bar{W}$ and Gaschütz's theorem yields that $\bar{K}_{2}^{\prime} \cap \bar{V}=\bar{W}$. Then, since $E W=W \times Z\left(W^{t}\right)$, again by Gaschütz's theorem we have $K_{2}^{\prime \prime} \cap V$ $=W$ and so $L_{2}=K_{2}^{\prime \prime} \times K_{2}^{\prime \prime}$.

(5.4) Set $G_{0}=\left\langle L_{1}, L_{2}\right\rangle$ and set $G_{1}=\left\langle K_{1}^{\prime}, K_{2}^{\prime \prime}\right\rangle$, where $K_{2}^{\prime \prime}$ is chosen such that $W \in \operatorname{Syl}_{2}\left(K_{1}^{\prime}\right) \cap \operatorname{Syl}_{2}\left(K_{2}^{\prime \prime}\right)$. Then $G_{0}=G_{1} \times G_{1}^{t}$ and $G_{1} \simeq U_{4}(q)$.

Proof. We will show $\left[K_{1}^{\prime t}, K_{2}^{\prime \prime}\right]=1$. We remark firstly that

$$
\begin{aligned}
F_{1}^{r_{2}} \cap F_{1}^{r_{2} r_{1} r_{2}} & =\left(F_{1} \cap F_{1}^{r_{2}}\right)^{r_{1} r_{2}}=\left(\left(W_{1} \times W_{1}^{t}\right) \cap\left(W_{1}^{r_{2}} \times W_{1}^{r_{2} t}\right)\right)^{r_{1} r_{2}} \\
& =\left(W_{1}^{r_{2}} \cap W_{1}^{r_{2} r_{1} r_{2}}\right) \times\left(W_{1}^{r_{2}} \cap W_{1}^{r_{2} r_{1} r_{2}}\right)^{t}
\end{aligned}
$$

and secondly by the structure of $A_{2} N_{2}=C_{L_{2}}(t)$ that

$$
A_{2} N_{2}=\left\langle P, A_{1}^{r_{2}} \cap A_{1}^{r_{2} r_{1} r_{2}}\right\rangle \text {. }
$$

Now by (5.3) the correspondence of $x$ to $x x^{t}$ for $x \in K_{2}^{\prime \prime}$ gives an isomorphism $K_{2}^{\prime \prime} \simeq C_{L_{2}}(t)$. Then the first remark shows that $W_{1}^{r_{2}} \cap W_{1}^{r_{2} r_{1} r_{2}}$ corresponds to $A_{1}^{r_{2}} \cap A_{1}^{r_{2} r_{1} r_{2}}$. Clearly $W$ corresponds to $P$. So by the second remark we have

$$
K_{2}^{\prime \prime}=\left\langle W, W_{1}^{r_{2}} \cap W_{1}^{r_{2} r_{1} r_{2}}\right\rangle .
$$

For $K_{1}^{\prime}$ as above we have

$$
\begin{aligned}
& F_{1}^{r_{2} r_{1}} \cap F_{2}^{r_{1}}=\left(W_{1}^{r_{2} r_{1}} \cap W_{2}^{r_{1}}\right) \times\left(W_{1}^{r_{2} r_{1}} \cap W_{2}^{r_{1}}\right)^{t}, \\
& K_{1}^{\prime} \simeq C_{L_{1}}(t)=A_{1} N_{1}=\left\langle P, A_{1}^{r_{2} r_{1}} \cap A_{2}^{r_{1}}\right\rangle,
\end{aligned}
$$

and

$$
K_{1}^{\prime}=\left\langle W, W_{1}^{r_{2} r_{1}} \cap W_{2}^{r_{1}}\right\rangle
$$

Then by (5.1.3) and (5.3)

$$
\left[W^{t}, W_{1}^{r_{2}} \cap W_{1}^{r_{2} r_{1} r_{2}}\right] \leq\left[W^{t}, W_{1}^{r_{2}}\right] \leq\left[K_{2}^{\prime \prime t}, K_{2}^{\prime \prime}\right]=1
$$

and

$$
\left[W,\left(W_{1}^{r_{2} r_{1}} \cap W_{2}^{r_{1}}\right)^{t}\right] \leq\left[W, W_{2}^{r_{1} t}\right] \leq\left[K_{1}^{\prime}, K_{1}^{\prime \prime}\right]=1 .
$$

Of course $\left[W, W^{t}\right]=1$. Moreover, since $\left(r_{1} r_{2}\right)^{4}=1$, we have 


$$
\begin{aligned}
{\left[W_{1}^{r_{2}}\right.} & \left.\cap W_{1}^{r_{2} r_{1} r_{2}},\left(W_{1}^{r_{2} r_{1}} \cap W_{2}^{r_{1}}\right)^{t}\right] \\
& \leq\left[W_{1}^{r_{2} r_{1} r_{2}}, W_{1}^{r_{2} r_{1} t}\right]=\left[W_{1}^{r_{2} r_{1} r_{2} r_{1}}, W_{1}^{r_{1} r_{2} r_{1} t}\right]=\left[W_{1}^{r_{2}}, W_{1}^{t}\right]^{r_{1} r_{2} r_{1}} \\
& \leq\left[K_{2}^{\prime \prime}, K_{2}^{\prime \prime}\right]^{r_{1} r_{2} r_{1}}=1 .
\end{aligned}
$$

Therefore we have $\left[K_{1}^{\prime t}, K_{2}^{\prime \prime}\right]=1$ and consequently $G_{0}=G_{1} * G_{1}^{t}$. Then $C_{G_{0}}(t)$ is a homomorphic image of $G_{1}$. Since $G_{1}$ is perfect, $G_{1}$ is a central extension of $U_{4}(q)$. Thus we have $G_{0}=G_{1} \times G_{1}^{t}$ and $G_{1} \simeq U_{4}(q)$.

(5.5) Let $S_{0}$ be a Sylow 2-subgroup of $N\left(F_{1}\right)$ containing $R$ and set $R_{0}=$ $N_{S_{0}}\left(K_{1}^{\prime}\right)$. Then

(1) $F_{1} \in \operatorname{Syl}_{2}\left(C\left(L_{1} / F_{1}\right)\right)$ and

(2) $S_{0} \in \operatorname{Syl}_{2}(G)$.

Proof. (1) is clear by the proof of (3.13.1), since $t \notin C\left(L_{1} / F_{1}\right)$. We will show that $F_{1}=\operatorname{Jr}\left(S_{0}\right)$ and then (2) follows.

Let $x \in I\left(S_{0}-R\right)$. Then by (1) $x$ acts on $L_{1} / F_{1}$ nontrivially. Hence $m\left(S_{0} / R\right) \leq 2$. If $x$ interchanges $K_{1}^{\prime}$ and $K_{1}^{\prime t}$, then $m\left(C_{R}(x)\right)=4 n$ and $m\left(C_{S_{0}}(x)\right)$ $\leq 4 n+2$. This implies $\operatorname{Jr}\left(S_{0}\right)=\operatorname{Jr}\left(R_{0}\right)$. Suppose $m\left(C_{C}\left(A_{2} N_{2} / A_{2}\right) / A_{2}\right)=1$. Then $C_{N\left(F_{2}\right) / F_{2}}\left(F_{2} N_{2} / F_{2}\right)$ has a cyclic Sylow 2-subgroup and $F_{2} N_{2} / F_{2}$ is a standard subgroup of $N\left(F_{2}\right) / F_{2}$. Hence we have $L_{2}=F_{2}\left\langle N_{2}^{N\left(F_{2}\right)}\right\rangle \triangleleft N\left(F_{2}\right)$ as in (3.13). Since $R=L_{1} \cap S_{0} \triangleleft S_{0}$ and $F_{2}=J r(R \bmod E)$ by (5.4), $S_{0}$ normalizes $L_{2}$. Hence $S_{0}$ normalizes $G_{0}$. Then we have $S_{0}<$ Aut $\left(G_{0}\right)$ and $R_{0}<N\left(G_{0}\right) \cap N\left(G_{1}\right)$. Thus (2) holds by $\left(2.3 .2,4\right.$ and 5). Consequently we may assume that $m\left(C_{C}\left(A_{2} N_{2}\right)\right.$ $\left.\left.A_{2}\right) / A_{2}\right)=2$, so $I(T-P)=I(t P) \cup I(u P) \cup I(t u P)$, where $u$ is defined in Section 2. We also may assume without loss of generality that $u$ acts on both components of $L_{1} / F_{1}$ as a field automorphism. Then $I(u P) \subseteq I\left(R_{0}\right)$ and $I(t P)$ $\cup I(t u P) \subseteq I\left(S_{0}-R_{0}\right)$. Since $F_{2}=J r(R \bmod E)$ and since $u$ normalizes $I_{2}$, it follows that $u \in N\left(L_{2}\right)$. Hence $u \in N\left(G_{0}\right)$. Then as above we have $\operatorname{Jr}(\langle u\rangle R)$ $=F_{1}$.

Therefore, if $I\left(R_{0}-R\right)=I(u R)$, then by the result of the previous paragraph there is nothing more to say. So we may assume that $m\left(R_{0} / R\right)=2$ and then we can choose an element $x$ of $R_{0}-\langle u\rangle R$ such that $x^{2} \in F_{1}, x$ acts on one of the components of $L_{1} / F_{1}$ trivially and on the other as a field automorphism. Suppose that $x$ acts on $K_{1} / F_{1}$ trivially. Let $z \in Z(W)$ and let $\Omega=z^{K_{1}}$. Then by (2.2.1) and (5.4) $|\Omega|=(q-1)\left(q^{2}+1\right)$. Since $\left(\langle x\rangle K_{1}\right)^{\Omega} \simeq Z_{2}$ $\times S L_{2}\left(q^{2}\right), x^{\Omega}$ is semiregular. Hence $x^{\Omega}=1^{\Omega}$, since $|\Omega|=$ odd. So we have $x \in C\left(W_{1}\right)$, since $\langle\Omega\rangle=W_{1}$. Then by (2.7) $x y \in C\left(K_{1}^{\prime}\right)$ for some $y \in W_{1}$. Set $s=(x y)^{t}$. Then

$$
K_{1}^{\prime}\langle s\rangle \cap K_{1}^{\prime t}\left\langle s^{t}\right\rangle \leq C\left(L_{1}\right) .
$$

By (1) $\left|C\left(L_{1}\right)\right|=$ odd. Hence we have

$$
K_{1}^{\prime}\langle s\rangle \cap K_{1}^{\prime t}\left\langle s^{t}\right\rangle=1 .
$$


So

$$
\begin{gathered}
L_{1}\left\langle s, s^{t}\right\rangle=K_{1}^{\prime}\langle s\rangle \times K_{1}^{\prime t}\left\langle s^{t}\right\rangle, \\
\Omega_{1}\left(R_{0} \bmod R\right)=R\left\langle s, s^{t}\right\rangle=W\langle s\rangle \times W^{t}\left\langle s^{t}\right\rangle
\end{gathered}
$$

and

$$
K_{1}^{\prime}\langle s\rangle \simeq C(t) \cap L_{1}\left\langle s, s^{t}\right\rangle=A_{1} N_{1}\langle u\rangle .
$$

Here we can choose $s$ to be an involution. Moreover we may assume that

$$
u=s s^{t} \text {. }
$$

Then for any involution $x$ of $s R$ we have $m\left(C_{K_{1}^{\prime}}(x)\right)=3 n$ by $(2.3 .4,5)$ and so $m\left(C_{R_{0}}(x)\right) \leq 7 n+1$. Therefore $\operatorname{Jr}\left(R_{0}\right)=\operatorname{Jr}(R)=F_{1}$. Thus the proof is complete.

(5.6) (1) The fusion of $F_{1}$ in $G$ is controlled by $\langle t\rangle J_{2} L_{1}$.

(2) $L_{2} \triangleleft N\left(F_{2}\right)$.

Proof. Set $\Omega=F_{1}^{*}$. Then by (2.2.1) and (5.4) $L_{1}^{\Omega}$ has two orbits of length $(q-1)\left(q^{2}+1\right)$ and $2(q-1)$ orbits of length $q\left(q^{2}+1\right)$ in $\left(W_{1} \cup W_{1}^{t}\right)^{\sharp}$, and one orbit of length $(q-1)^{2}(q+1)^{2}, 2(q-1)$ orbits of length $(q-1) q\left(q^{2}+1\right)^{2}$ and $(q-1)^{2}$ orbits of length $q^{2}\left(q^{2}+1\right)^{2}$ in $F_{1}-\left(W_{1} \cup W_{1}^{t}\right)$. Also we have that $\left(\langle t\rangle J_{2} L_{1}\right)^{\Omega}$ permutes transitively the orbits of $L_{1}^{\Omega}$ of the same length, that is, $\left(\langle t\rangle J_{2} L_{1}\right)^{2}$ has five orbits which are of length $2(q-1)\left(q^{2}+1\right), 2(q-1) q\left(q^{2}+1\right)$ in $\left(W_{1} \cup W_{1}^{t}\right)^{\#}$ and $(q-1)^{2}\left(q^{2}+1\right)^{2}, 2(q-1)^{2} q\left(q^{2}+1\right)^{2},(q-1)^{2} q^{2}\left(q^{2}+1\right)^{2}$ in $F_{1}-\left(W_{1} \cup W_{1}^{t}\right)$. Since $F_{1}$ is weakly closed in $S_{0}$, the fusion of the elements of $F_{1}$ is controlled by $N\left(F_{1}\right)$, which permutes the orbits of $L_{1}^{\Omega}$ of the same length. Hence the fusion is already controlled by $\langle t\rangle J_{2} L_{1}$.

Then $W_{2} \cup W_{2}^{t}$ is weakly closed in $F_{2}$ with respect to $G$, since $F_{2}^{*} \cap W_{1}^{G}=$ $I\left(W_{2} \cup W_{2}^{t}\right)$ and $\left\langle I\left(W_{2}\right)\right\rangle=W_{2}$. Therefore, if we set $X=N\left(F_{2}\right) \cap N\left(W_{2}\right)$, then $\left|N\left(F_{2}\right): X\right|=2$ and $R_{0} \in \operatorname{Syl}_{2}(X)$. Let $Y=\Omega_{1}\left(R_{0} \bmod R\right)$. In order to prove $L_{2} \triangleleft N\left(F_{2}\right)$ we may assume that $m\left(C_{C}\left(A_{2} N_{2} / A_{2}\right) / A_{2}\right)=2$ as in the proof of (5.5). Hence we have

$$
\begin{aligned}
& Y=\langle u\rangle R \text { if } m\left(R_{0} / R\right)=1, \text { or } \\
& Y=\left\langle s, s^{t}\right\rangle R=W\langle s\rangle \times W^{t}\left\langle s^{t}\right\rangle \text { if } m\left(R_{0} / R\right)=2,
\end{aligned}
$$

where $s$ is chosen as in (5.5). In any case $\left[R / F_{2}, s\right]=\left[R / F_{2}, u\right]=1$ by (2.5.3). So we have

$$
Y=J r\left(R_{0} \bmod F_{2}\right)=\Omega_{1}\left(R_{0} \bmod F_{2}\right)
$$

and also $Y / F_{2}$ is abelian. Therefore the fusion of $I\left(R_{0} / F_{2}\right)$ in $X$ is controlled by $N_{X}(Y)$. Since $Y>F_{1}=\operatorname{Jr}\left(S_{0}\right)$, we have 


$$
N_{X}(Y) \leq N_{X}\left(F_{1}\right)=N_{X}(R),
$$

which implies that $N_{X}(R)$ controls the fusion of $I\left(R_{0} / F_{2}\right)$ in $X$. Consequently any involution of $R_{0}-R$ does not fuse into $R$ in $X$. Suppose that $u^{x} \in s R$ for some $x \in X$. Then we may take $x \in N_{X}(R)$. But $\langle s\rangle R \neq\langle u\rangle R$. This gives a contradiction. Hence $u^{X} \subseteq u R$. Since $R_{0} / R$ is abelian, we have $u \notin O^{2}(X)$ by (1.5). Suppose $R \notin \operatorname{Syl}_{2}\left(O^{2}(X)\right)$. Then $R\langle s\rangle$ or $R\left\langle s^{t}\right\rangle\left\langle O^{2}(X)\right.$, since $R<L_{2}$ $\leq O^{2}(X)$. This yields that $u \in R\left\langle s, s^{t}\right\rangle \leq O^{2}(X)$, since $O^{2}(X)$ is $t$-invariant, a contradiction. Hence $R \in \operatorname{Syl}_{2}\left(O^{2}(X)\right)$. Then $F_{2} N_{2} / F_{2}$ is a standard subgroup of $O^{2}(X)\langle t\rangle \mid F_{2}$ and $\langle t\rangle F_{2} / F_{2}$ is a Sylow 2-subgroup of $C\left(F_{2} N_{2} / F_{2}\right) \cap O^{2}(X)\langle t\rangle / F_{2}$. Hence as in (4.9) we have $L_{2} \triangleleft N\left(F_{2}\right)$.

(5.7) (1) $N\left(F_{i}\right) \leq N\left(G_{0}\right), i=1,2$.

(2) $N\left(G_{0}\right)$ controls the $G$-fusion of involutions of $G_{0}$.

(3) If $t \in N\left(G_{0}\right)^{\text {g }}$, then $g \in N\left(G_{0}\right)$.

(4) $\left|C\left(G_{0}\right)\right|=$ odd.

Proof. (4) holds obviously by (5.5.1). Since $L_{i} \triangleleft N\left(F_{i}\right)$ for $i=1$ and 2, the Frattini argument yields that $N\left(F_{i}\right)=L_{i}\left(N(R) \cap N\left(F_{i}\right)\right)$. By (5.4) $F_{2}=$ $J r(R \bmod E)$. Hence $F_{i}$ char $R, i=1,2$. Therefore $N\left(F_{i}\right)=L_{i}\left(N\left(F_{3-i}\right) \cap N\left(F_{i}\right)\right)$, which normalizes $G_{0}$. Thus (1) holds. By (2.4) and (5.4) any involution of $G_{0}$ fuses into $F_{1}$. Hence (2) follows from (1) and (5.6.1).

By (1) $S_{0}<$ Aut $\left(G_{0}\right)$. If an involution $x$ of $S_{0}$ does not interchange $G_{1}$ and $G_{1}^{t}$, then by (2.3.4) $m(C(x)) \geq 6 n+1$. Since $m(C)=4 n+1, t$ interchanges $G_{1}^{g}$ and $G_{1}^{t g}$, whenever $t \in N\left(G_{0}\right)^{g}$. Hence $C(t) \cap G_{0}^{g}=\left\{x x^{t} \mid x \in G_{1}^{g}\right\}$ and $L \simeq C(t)$ $\cap C_{0}^{g} \leq C^{(\infty)}=L$. Therefore we have $C(t) \cap G_{0}^{g}=L$. Let $R^{\prime}\langle t\rangle$ be a Sylow 2subgroup of $G_{0}\langle t\rangle$ containing $Q$. Then $N_{R^{\prime}\langle t\rangle}(Q)>Q$. Hence by Lemma 2.5.3 of [2] we have $\left\langle N_{R^{\prime}}(Q), L\right\rangle=G_{0}^{g}$. On the other hand by (3.2.2) and (3.3) $N(Q)=E N_{C}(Q) \leq N(R)$. Hence $N(Q) \leq N\left(Q_{0}\right)$ and consequently $G_{0}^{g} \leq N\left(G_{0}\right)$. Then $G_{0}^{g} \leq N\left(G_{0}\right)^{(\infty)}=G_{0}$ and clearly the equality holds.

(5.8) There exists an abelian subgroup $R_{1}$ of $N\left(G_{0}\right) \cap N\left(G_{1}\right)$ such that $R \cdot R_{1} \in \operatorname{Syl}_{2}\left(N\left(G_{0}\right) \cap N\left(G_{1}\right)\right)$, $t$ normalizes $R_{1}$ and $C_{R_{1}}(t)$ acts on $G_{1}$ and $G_{1}^{t}$ as a group of field automorphisms.

Proof. By (5.7.1) $S_{0}<N\left(G_{0}\right)$ and $R_{0} \in \operatorname{Syl}_{2}\left(N\left(G_{0}\right) \cap N\left(G_{1}\right)\right)$. Set $N\left(G_{0}\right)$

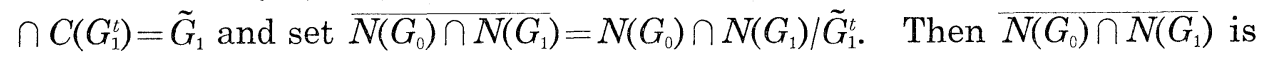
embedded in Aut $\left(U_{4}(q)\right)$. Hence there exists an element $\bar{x}$ which is a generator of the group of field automorphisms in $N\left(G_{0}\right) \cap N\left(G_{1}\right)$ and which normalizes $\bar{R}$. Let $x$ be a preimage of $\bar{x}$ and set $X=\tilde{G}_{1}^{t}\langle x\rangle \cap \tilde{G}_{1}\left\langle x^{t}\right\rangle$. Notice that $G_{1} \leq \tilde{G}_{1}$ and $G_{1} \tilde{G}_{1}^{t}\langle x\rangle=N\left(G_{0}\right) \cap N\left(G_{1}\right)$. Hence we have

$$
G_{0} X=G_{1}^{t}\left(G_{1} \tilde{G}_{1}^{t}\langle x\rangle \cap \tilde{G}_{1}\left\langle x^{t}\right\rangle\right)=G_{1} \tilde{G}_{1}^{t}\langle x\rangle \cap G_{1}^{t} \tilde{G}_{1}\left\langle x^{t}\right\rangle=N\left(G_{0}\right) \cap N\left(G_{1}\right)
$$


and

$$
G_{0} \cap X=G_{0} \cap \tilde{G}_{1}^{t}\langle x\rangle \cap \tilde{G}_{1}\left\langle x^{t}\right\rangle=G_{1}^{t} \cap \tilde{G}_{1}\left\langle x^{t}\right\rangle=1 .
$$

Therefore $N\left(G_{0}\right) \cap N\left(G_{1}\right)=G_{0} \cdot X$ and by the choice of $x X \cap C$ acts on $G_{1}$ and $G_{1}^{t}$ as a group of field automorphisms. Since

$$
R \tilde{G}_{1}^{t} \cap R \tilde{G}_{1}=R\left(W \tilde{G}_{1}^{t} \cap \tilde{G}_{1}\right)=R\left(\tilde{G}_{1}^{t} \cap \tilde{G}_{1}\right)=R \times C\left(G_{0}\right),
$$

$X$ normalizes $R$. Thus if we choose a $t$-invariant Sylow 2-subgroup $R_{1}$ of $X$, then we have $R \cdot R_{1} \in \operatorname{Syl}_{2}\left(N\left(G_{0}\right) \cap N\left(G_{1}\right)\right)$. By (5.7.4) $R_{0} / R$ is abelian. Therefore $R_{1}$ is abelian.

(5.9) Let $\psi$ be the transfer homomorphism of $G$ into $S_{0} / R_{0}$ relative to the subgroup $S_{0}$. Then

(1) $t, t u \notin \operatorname{Ker} \psi$ and

(2) $u^{G} \cap S_{0} \subseteq u R$.

Proof. By the structure of $G_{0}$,

$$
m\left(C_{N\left(G_{0}\right)}(x)\right) \geq 6 n+1 \quad \text { for } x \in I(R) .
$$

Hence $t^{G} \cap R_{0}=\phi$. Suppose $(t u)^{g} \in R_{0}$ for some $g \in G$. Since

$$
m\left(C_{N\left(G_{0}\right)}(t u)\right)=4 n+1,
$$

we can choose $C_{S_{0}}\left((t u)^{g}\right)$ to be a Sylow 2-subgroup of $C\left((t u)^{g}\right)$. Furthermore we may assume that $C_{S_{0}}(t u)^{g} \leq C_{S_{0}}\left((t u)^{g}\right)$. Then $t^{g}, t \in S_{0}$. So by (5.7.3) $g \in N\left(G_{0}\right)$ and $(t u)^{g} \notin R_{0}$, which is a contradiction. Thus (1) holds.

we have

By (5.8) $\Omega_{1}\left(R_{0}\right)=\langle u\rangle R$ or $\left\langle s, s^{t}\right\rangle R$ according as $m\left(R_{0} / R\right)=1$ or $2 . \quad$ By (2.8)

$$
\begin{aligned}
& m\left(C_{S_{0}}(u)\right) \leq 6 n+2 \text { and } \\
& m\left(C_{N\left(G_{0}\right)}(x)\right) \geq 7 n+1 \text { for } x \in I(\langle s\rangle R) \cup I\left(\left\langle s^{t}\right\rangle R\right) .
\end{aligned}
$$

Therefore if $u^{g} \in\langle s\rangle R \cup\left\langle s^{t}\right\rangle R$ for some $g \in G$, then we may take $C_{S_{0}}(u)^{g}$ $\leq C_{S_{0}}\left(u^{g}\right), t^{g}, t \in N\left(G_{0}\right)$ and we have $g \in N\left(G_{0}\right)$ as above. This does not occur. Thus $u^{G} \cap R_{0} \subseteq u R$.

Now in order to prove (2) we will show $u^{G} \cap\left(S_{0}-R_{0}\right)=\phi$. Take involutions $w_{1}$ and $w_{2}$ of $W$ such that $w_{1} w_{1}^{t}=z_{1}$ and $w_{2} w_{2}^{t}=z_{2}$. Then by (5.7.2) the conjugacy classes of involutions of $G_{0}$ in $G$ are represented by $w_{1}, w_{2}, z_{1}, z_{2}$ and $w_{1} w_{2}^{t}$. By $(2.8) m\left(C_{W}(x)\right)=m\left(C_{W t}(x)\right)=3 n$ for any involution $x$ of $u R$. Hence we have

$$
m\left(C_{S_{0}}(x)\right) \geq 6 n+1,
$$

while 


$$
m(B) \leq 3 n+2,
$$

where $B$ is an elementary abelian subgroup of $C_{S_{0}}(x)$ such that

$$
B^{\sharp} \subseteq z_{1}^{G} \cup z_{2}^{G} \cup\left(w_{1} w_{2}^{t}\right)^{G} .
$$

On the other hand $m\left(C_{R}(x)\right)=4 n$ for any involution $y$ of $S_{0}-R_{0}$, since $R=$ $W \times W^{t}=W \times W^{y}$. Hence we have

$$
m\left(C_{S_{0}}(y)\right) \leq 4 n+2, \quad \text { and } \quad m\left(C(y) \cap W_{1} W_{1}^{y}\right)=4 n .
$$

Note that

$$
\left(C(y) \cap W_{1} W_{1}^{t}\right)^{\sharp} \subseteq z_{1}^{G} \cup z_{2}^{G} \cup\left(w_{1} w_{2}^{t}\right)^{G} .
$$

These facts imply that any involution of $S_{0}-R_{0}$ does not fuse into $u R$ if $n>2$. Hence by the result of the second paragraph we have $u^{G} \cap S_{0} \subseteq u R$, if $q>4$.

Suppose that $q=4$ and $u$ fuses into $S_{0}-R_{0}$. Choose a conjugate $u^{\prime}$ of $u$ such that $C_{S_{0}}\left(u^{\prime}\right) \in \operatorname{Syl}_{2}\left(C\left(u^{\prime}\right)\right)$ and choose $u^{\prime \prime}$ such that $u^{\prime \prime} \in u^{G} \cap\left(S_{0}-R_{0}\right)$. We may assume that $u^{\prime \prime g}=u^{\prime}$ and $C_{S_{0}}\left(u^{\prime \prime}\right)^{g} \leq C_{S_{0}}\left(u^{\prime}\right)$ for some $g \in G$. Set $B^{\prime \prime}=$ $\operatorname{Jr}\left(C_{R}\left(u^{\prime \prime}\right)\right)$ and set $B^{\prime}=B^{\prime \prime g}$. The result of the second paragraph gives that $u^{\prime} \in u R$. Then by (2.6) $C_{W}\left(u^{\prime}\right) \simeq C_{W^{t}}\left(u^{\prime}\right)$ is isomorphic to a Sylow 2-subgroup of $\operatorname{Sp}_{4}(q)$. So, if we set $W_{i+2}=C_{W_{i}}\left(u^{\prime}\right)$ for $i=1,2$ and take $t^{\prime} \in t^{G} \cap C_{S_{0}}\left(u^{\prime}\right)$, then

$$
\mathscr{E}\left(C_{R}\left(u^{\prime}\right)\right)=\left\{W_{3} W_{3}^{t^{\prime}}, W_{3} W_{4}^{t^{\prime}}, W_{4} W_{3}^{t^{\prime}}, W_{4} W_{4}^{t^{\prime}}\right\}
$$

and

$$
m\left(W_{3}\right)=m\left(W_{4}\right)=3 n
$$

Suppose that $B^{\prime} \leq R_{0}$. Then $m\left(C_{S_{0}}\left(u^{\prime}, b\right)\right)=4 n$ whenever $b \in B^{\prime}-R_{0}$. But $B^{\prime \prime}\left\langle u^{\prime \prime}\right\rangle \leq C_{S_{0}}\left(u^{\prime \prime}, B^{\prime \prime}\right)$ which gives that $m\left(C_{S_{0}}\left(u^{\prime \prime}, B^{\prime \prime}\right)\right)=4 n+1$. This contradicts the choice of $u^{\prime}$. Hence $B^{\prime} \leq R_{0}$. Since $\Omega_{1}\left(R_{0}\right)=\langle s\rangle W \times\left\langle s^{t}\right\rangle W^{t}$, there exists a unique element $s^{\prime}$ in $s W$ such that $s^{\prime} u^{\prime} \in s^{t} W^{t}$. Then for some $x, y \in C_{R}\left(u^{\prime}\right)$ we have

$$
B^{\prime}=\left(B^{\prime} \cap R\right)\left\langle s^{\prime} x\right\rangle\left\langle s^{\prime} u^{\prime} y\right\rangle .
$$

Now by focal subgroup theorem, $m\left(R_{0} \cap O^{2}(G) / R\right)=2$. Then it is clear that $m\left(C_{R_{0}}\left(u^{\prime \prime}\right) \cap O^{2}(G) / C_{R}\left(u^{\prime \prime}\right)\right)=1$. Therefore

$$
\left|C_{S_{0}}\left(u^{\prime \prime}\right) \cap O^{2}(G) / C_{S_{0}}\left(u^{\prime \prime}, B^{\prime \prime}\right) \cap O^{2}(G)\right| \geq 2 q^{2} .
$$

Next we calculate $\left|C_{S_{0}}\left(u^{\prime}\right) \cap N\left(B^{\prime}\right) / C_{S_{0}}\left(u^{\prime}, B^{\prime}\right)\right|$. Suppose that $B^{\prime}<W_{i^{\prime}} W_{j^{\prime}}^{t^{\prime}}$ for any $i^{\prime}, j^{\prime} \in\{3,4\}$. Then $C_{R}\left(u^{\prime}, B^{\prime} \cap R\right)=W_{i^{\prime}} W_{j^{\prime}}^{t^{\prime}}$ in which $x$ and $y$ lie. Hence we have $C_{R}\left(u^{\prime}, B^{\prime}\right)=W_{i^{\prime}} W_{j^{\prime}}^{t^{\prime}}$ and so

$$
\left|N_{R}\left(R \cap B^{\prime}\right) \cap C_{R}\left(u^{\prime}\right) / C_{R}\left(u^{\prime}, B^{\prime}\right)\right|=q .
$$


Hence

$$
\left|N_{S_{0}}\left(B^{\prime}\right) \cap C_{S_{0}}\left(u^{\prime}\right) \cap O^{2}(G) / C_{S_{0}}\left(u^{\prime}, B^{\prime}\right) \cap O^{2}(G)\right| \leq 4 q \leq 2 q^{2} .
$$

Again this contradicts the choice of $u^{\prime}$. Thus $u$ does not fuse into $S_{0}-R_{0}$, even if $q=4$.

(5.10) $R \in \operatorname{Syl}_{2}\left(O^{2}(G)\right)$.

Proof. We may assume that $R_{0}=R \cdot R_{1}$ by (5.8). We will show that any element $x$ of $R_{1}\langle t\rangle-R$ does not fuse into $R_{0}$, and that any element $y$ of $R_{1}$ such that $\Omega_{1}(\langle y\rangle)<C$ does not fuse into $S_{0}-R_{0}$. These facts imply that $R_{0} \in \mathrm{Syl}_{2}(\operatorname{Ker} \psi)$.

Suppose that $|x|>2$. Then $x=x_{1} t$ for some $x_{1} \in R_{1}$ and $x^{2}=x_{1} x_{1}^{t} \in C$. Hence $I(\langle x\rangle)$ is conjugate to $u$. Therefore for any $g \in G$ such that $x^{g} \in S_{0}$ we have $\left|x^{g} R / R\right|=|x|$ by (5.9.2). Hence if $x^{g} \in R_{0}$, then by (2.8)

$$
\begin{aligned}
& C_{R}\left(x^{g}\right)=C_{W}\left(x^{g}\right) \times C_{W^{t}}\left(x^{g}\right) \text { and } \\
& m\left(C_{W}\left(x^{g}\right)\right)=m\left(C_{W^{t}}\left(x^{g}\right)\right)=3 m, \text { where } m=2 n /|x| .
\end{aligned}
$$

Next if $x^{h} \in S_{0}-R_{0}$ for some $h$, then

$$
C_{R}\left(x^{h}\right)=\left\{w w^{x h} \mid w \in C_{W}\left(x^{2 h}\right)\right\} .
$$

Now for a subgroup $X$ of $G$ we define the following:

$$
\begin{aligned}
& m_{1}(X)=\operatorname{Max}\left\{m(B) \mid B \leq X, B^{\sharp} \subseteq z_{1}^{G} \cup z_{2}^{G} \cup\left(w_{1} w_{2}^{t}\right)^{G}\right\} ; \\
& m_{2}(X)=\operatorname{Max}\left\{m\left(B^{\prime}\right) \mid B^{\prime} \leq X, B^{\prime \#} \subseteq w_{1}^{G} \cup w_{2}^{G}\right\} .
\end{aligned}
$$

Then the above result gives that

$$
\begin{aligned}
& m_{1}\left(C_{R}\left(x^{g}\right)\right)=3 m, m_{2}\left(C_{R}\left(x^{g}\right)\right)=3 m, \\
& m_{1}\left(C_{R}\left(x^{h}\right)\right)=6 m, m_{2}\left(C_{R}\left(x^{h}\right)\right)=0 .
\end{aligned}
$$

Therefore we have

$$
\begin{aligned}
& m_{1}\left(C_{S_{0}}\left(x^{g}\right)\right) \leq 3 m+2, m_{2}\left(C_{S_{0}}\left(x^{g}\right)\right) \geq 3 m, \\
& m_{1}\left(C_{S_{0}}\left(x^{h}\right)\right) \geq 6 m, m_{2}\left(C_{S_{0}}\left(x^{h}\right)\right) \leq 2 .
\end{aligned}
$$

Consequently for any $x^{g} \in R_{0}$ and any $x^{h} \in S_{0}-R_{0}$,

$$
\begin{aligned}
& m_{1}\left(C_{S_{0}}\left(x^{g}\right)\right)<m_{1}\left(C_{S_{0}}\left(x^{h}\right)\right) \\
& m_{2}\left(C_{S_{0}}\left(x^{g}\right)\right)>m_{2}\left(C_{S_{0}}\left(x^{h}\right)\right),
\end{aligned}
$$

which is absurd, since $C_{S_{0}}\left(x^{\prime}\right) \in \operatorname{Syl}_{2}\left(C\left(x^{\prime}\right)\right)$ for some conjugate $x^{\prime}$ of $x$ in $S_{0}$. Thus $x$ does not fuse into $R_{0}$. Similarly we can obtain that $y$ does not fuse 
into $S_{0}-R_{0}$. Therefore $x \notin \operatorname{Ker} \psi$ for any $x$ of $t R_{1}$ of order at least 4 . Let $R_{1}^{\prime}=R_{1}\langle t\rangle \cap \operatorname{Ker} \psi$. Suppose $R_{1}^{\prime} \neq R_{1}$. Then any element of $R_{1}^{\prime}-R$ is an involution. This yields that $R_{1}^{\prime} \cap R_{1}$ is cyclic and so $R_{1}^{\prime}$ is a dihedral group by the structure of Aut $\left(G_{0}\right)$. Since $t \notin R_{1}^{\prime}, R_{1}^{\prime}\langle t\rangle$ is also dihedral and so $R_{1}$ is cyclic. Then $\Omega_{1}(\langle y\rangle)<C$ for any $y$ of $R_{1}$. Since such elements do not fuse into $S_{0}-R_{0}$, we have $R_{1}<\operatorname{Ker} \psi$, a contradiction. Therefore $R_{1}^{\prime}=R_{1}$ and $R_{0}=S_{0} \cap \operatorname{Ker} \psi$. Then, since $R_{0} / R$ is abelian, $u \notin O^{2}(G)$ by (5.9.2), and we can obtain the assertion as in the proof of (5.9.2).

For the argument in $(5.11,12)$ below we refer the readers to $4.23,24$ of [21].

(5.11) (1) $W_{1}$ and $W_{1}^{t}$ are weakly closed in $R$ with respect to $O^{2}(G)$.

(2) $W \in \operatorname{Syl}_{2}\left(O^{2}\left(C_{Q}\left(W_{1}^{t}\right)\right)\right)$.

Proof. By (5.7.2), if $W_{1}^{g}<R$, then $W_{1}^{g} \subseteq W \cup W^{t}$. If $W_{1}^{g}<W$, then $W_{1}^{g}=$ $W_{1}$ by (2.3.2). If $W_{1}^{g}<W^{t}$, then $W_{1}^{g}=W_{1}^{t}$. So, if $W_{1}^{g}=W_{1}^{t}$, then $N(R) \cap O^{2}(G)$ permutes $W_{1}$ and $W_{1}^{t}$ by a theorem of Burnside. But this can not occur, since $R \in \operatorname{Syl}_{2}\left(O^{2}(G)\right)$. Thus (1) holds. It is clear that $W \times W_{1}^{t} \in \operatorname{Syl}_{2}\left(C\left(W_{1}^{t}\right)\right.$ $\left.\cap O^{2}(G)\right)$. Hence by Gaschütz's theorem we have $W \in \operatorname{Syl}_{2}\left(O^{2}\left(C_{G}\left(W_{1}^{t}\right)\right)\right)$.

$$
\text { (5.12) } G_{0} \unlhd G \text {. }
$$

Proof. First we will show that $W$ and $W^{t}$ are strongly closed in $R$ with respect to $O^{2}(G)$. Let $x \in W$ and suppose that $x^{g} \in R-W$ for some $g \in O^{2}(G)$. If $x$ is an involution, then by (5.6.1) we may take $x \in W_{1}$ and $x^{g} \in W_{1}^{t}$. Since $F_{1}=J r(R), x$ and $x^{g}$ are conjugate in $N\left(F_{1}\right) \cap O^{2}(G)$. But this contradicts (5.11.1).

Next we assume that $|x|=2^{i}, i \geq 2$. Set $x^{g}=x_{1} x_{2}$, where $x_{1} \in W$ and $x_{2} \in W^{t}$. If $\left|x_{1}\right| \leq\left|x_{2}\right|$, then $x^{2^{i-1}}$ which is an involution in $W$ is conjugate to $\left(x^{g}\right)^{2^{i-1}}$ which is an involution in $R-W$. This contradicts the first paragraph. Hence $\left|x_{1}\right|>\left|x_{2}\right|$, and replacing $x$ by an appropriate power, we may assume $\left|x_{2}\right|=2$. Furthermore we may assume that $x_{1} \in W$ and $x_{2} \in W_{1}^{t}$ by conjugation in $G_{0}$. Then $x, x^{g} \in C\left(W_{1}^{t}\right)$. Hence by (5.11.2) we have $x \in O^{2}\left(C\left(W_{1}^{t}\right)\right)$ and $x^{g} \notin O^{2}\left(C\left(W_{1}^{t}\right)\right)$. Then (5.11.1) gives a contradiction. Thus $W$ is strongly closed in $R$ with respect to $O^{2}(G)$. By symmetry $W^{t}$ is also strongly closed.

Now we are in a position to quote a result of Goldschmidt [11] in order to obtain the final result of this section Set $X=\left\langle W^{o^{2(G)}}\right\rangle$. Then by [11] we have $\left[X, X^{t}\right]=1$, since we are assuming $O(G)=1$. Then $\left\{x x^{t} \mid x \in X\right\}$ is a homomorphic image of $X$ contained in $C$, and the kernel is contained in $X \cap X^{t} \leq Z(X)$. Therefore $X^{(\infty)} \simeq U_{4}(q)$. Since $\left\langle W^{G_{0}}\right\rangle=G_{1}$, we have $G_{0}=X X^{t}$. Thus $G_{0} \unlhd G$. 


\section{§6. Proof of the theorem}

We have assumed in Section 4 and 5 that $O(G)=1$. Now we will exclude this condition. Let $X$ denote the normal closure of $L$ in $G$ and let $\bar{G}=G / O(G)$. Then $\bar{L}$ is a standard subgroup of $\bar{G}$ whose centralizer has a cyclic Sylow 2subgroup. So we have either $\bar{L} \unlhd \bar{G}$ or $\bar{X} \simeq L_{4}\left(q^{2}\right)$ or $U_{4}(q) \times U_{4}(q)$ by the results of Section 4 and 5 . If one of the latter two cases holds, then in each case we can easily find a $t$-invariant 2-subgroup $A$ of $X$ such that $1 \neq[A, t]$ $\leq L$. Then by $(1 J)$ of [12] we have $[A, t, O(G)]=1$. Hence in particular $C_{X}(O(X)) \not \subset(X)$. Thus $O(X)=Z(X)$ and the theorem is established.

\section{References}

[1] M. Aschbacher, $\mathscr{F}$-setts and permutation groups, J. Algebra, 30 (1974), 400-416.

[2] M. Aschbacher, On finite groups of component type, Illinois J. Math., 19 (1975), 87-115.

[3] M. Aschbacher, A characterization of certain Chevalley groups and its application to component type groups, to appear.

[4] M. Aschbacher and G. Seitz, Involutions in Chevalley groups over fields of even order, to appear.

[5] M. Aschbacher and G. Seitz, On groups with a standard component of known type, to appear.

[6] A. Borel, R. Carter, C. W. Curtis, N. Iwahori, T. A. Springer and R. Steinberg, Seminar on algebraic groups and related finite groups, Lecture Notes in Mathematics, 3 (1970), Springer.

[7] R. Carter, Simple groups of Lie type, John Wiley and Sons, London, 1972.

[8] P. Fong and G. Seitz, Groups with a $(B, N)$-pair of rank 2, I, Invent. Math., 21 (1973), 1-57.

[ 9 ] G. Glauberman, Central elements in core-free groups, J. Algebra, 4 (1966), 403420.

[10] D. Goldschmidt, 2-fusion in finite groups, Ann. of Math., 99 (1974), 70-117.

[11] D. Goldschmidt, Strongly closed 2-subgroups of finite groups, Ann. of Math., 102 (1975) , 475-489.

[12] K. Gomi, Finite groups with a standard subgroup isomorphic to $\operatorname{Sp}\left(4,2^{n}\right)$, Japan. J. Math., 4 (1978), 1-76.

[13] D. Gorenstein, Finite groups, Harper and Row, New York, 1968.

[14] D. Gorenstein and J. Walter, Centralizers of involutions in balanced groups, J. Algebra, 20 (1972), 284-319.

[15] R. Griess, Schur multipliers of finite simple groups of Lie type, Trans. Ames. Math. Soc., 183 (1973), 355-421.

[16] R. Griess D. Mason and G. Seitz, Bender group as standard subgroups, to appear.

[17] G. Seitz, Chevalley groups as standard subgroups, to appear.

[18] R. Steinberg, Générateus, relations et revétements de groups algébriques, Colloquium sur la théorie des groups algébriques (Bruxelles) (1962), 113-127.

[19] F. Timmesfeld, Groups with weakly closed T. I. subgroups, Math. Z., 143 (1975), 243-278.

[20] H. Yamada, Finite groups with a standard subgroup isomorphic to $G_{2}\left(2^{n}\right)$, to appear. 
[21] L. Finkelstein, Finite groups with a standard component isomorphic to $M_{23}$, J. Algebra, 40 (1976), 541-555.

\section{Department of Computer Science \\ YAMANASHI UNIVERSITY \\ TAKEDA KOFU 400}

JAPAN 